\title{
Non-isotropic Gevrey Hypoellipticity for Grushin Operators
}

\author{
By \\ Yoshiaki Hashimoto, Takaaki Hoshino** and Tadato Matsuzawa***
}

\begin{abstract}
We shall determine non-isotropic Gevrey exponents for general Grushin operators based on the results given in the paper [26], where a method to determine isotropic (worst) Gevrey exponents was given. The ideas of the bracket calculus given in the paper [2] and FBI-transformation given in the paper [5] are also useful.
\end{abstract}

\section{$\S 1$. Introduction}

In the early 70s, V. V. Grushin has introduced a wide class of degenerate elliptic differential operators which are hypoelliptic in a series of the papers [10], [11] and [12]. After then, there has been investigated the problem of analytic and non-analytic hypoellipticity of the Grushin operators [1], [2], [13], [28], etc.

In the paper [26], we have tried to determine isotropic (equi-directional) Gevrey exponent of hypoellipticity for every Grushin operator. Our method given there is based on the Grushin's idea using operator-valued pseudodifferential operators [12] and our results on Gevrey calculus for pseudodifferential operators [22], [27].

Communicated by T. Kawai. Received October 30, 2000. Revised May 9 and August $29,2001$.

2000 Mathematics Subject Classification(s): 34, 35, 46.

Key words and phrases: Gevrey hypoellipticity, Gevrey exponents, Degenerate ellptic operators, Pseudodifferential operators, FBI-transformation.

*Institute of Natural Sciences, Nagoya City University, Nagoya 467-8501, Japan.

e-mail: hashimot@nsc.nagoya-cu.ac.jp

**High School attached to Meijo University, Nagoya 453-0031, Japan.

e-mail: thtiga1@pop16.odn.ne.jp

*** Department of Mathematics, Meijo University, Nagoya 468-8502, Japan.

e-mail: tadato@meijo-u.ac.jp 
In general, we know that hypoelliptic operators may have different Gevrey exponents with respect to different variables (directions). There has been remained open the problem to determine non-isotropic (directional) Gevrey exponents precisely for each Grushin operator.

Meanwhile, by using the method of bracket calculus, A. Bove and D. Tartakoff [2] succeeded to determine precise non-isotropic Gevrey exponents for generalized Baouendi-Goulaouic operators:

$$
P=\partial_{y}^{2}+y^{2 k} \partial_{x}^{2}+y^{2 l} \partial_{z}^{2}, \quad(k \geq l \geq 0, k>0, \text { see Example (c) in Section } 2) .
$$

They have proved that the operator $P$ has $G_{x, z, y}^{\{\theta, 1, d\}}$-hypoellipticity in a neighborhood of the origin, where $\theta=(1+k) /(1+l)$ and $d=(\theta+k) /(1+k)$. Here we have $1<d<\theta$. This means the operator $P$ is analytic hypoelliptic with respect to $z$ but not $y$. The above operator $P$ is considered to be a typical Grushin operator as well as that of L. Hörmander [17]. Their idea using bracket calculus will be also useful in this paper.

In the paper [26], we have treated Grushin operators dividing them into three groups. In this paper, we would like to treat them also dividing into three groups. We shall start from the assumption that $C^{\infty}$-hypoellipticity is already proved for the Grushin operators. The other typical Grushin operators than $P$ are given by

$$
\begin{aligned}
L & =\partial_{y}^{2}+\left(x^{2 l}+y^{2 k}\right) \partial_{x}^{2}, \quad(l, k,=1,2, \ldots) \\
M & =\partial_{y}^{2}+\left(x^{2 l}+y^{2 k}\right)\left(\partial_{x}^{2}+\partial_{z}^{2}\right), \quad(l, k=1,2, \ldots) .
\end{aligned}
$$

The operator $L$ is $G_{x, y}^{\{\theta, d\}}$-hypoelliptic in a neighborhood of the origin in $\mathbf{R}^{2}$, where $\theta=(l(1+k)) /(l(1+k)-k)$ and $d=(\theta+k) /(1+k)$. The optimality of this exponent $\{\theta, d\}$ was already shown in the paper [28]. While, in Section 6 the operator $M$ will be proved having $G_{x, z, y}^{\{\theta, 1, d\}}$-hypoellipticity in a neighborhood of the origin in $\mathbf{R}^{3}$, where $\theta=(l(1+k)) /(l(1+k)-k)$ and $d=(\theta+k) /(1+k)$, (cf. Theorem 2.1 and Examples). Note that we have also $1<d<\theta$, for both operators $L$ and $M$. The precise definition of the Gevrey spaces will be given in Section 2.

In this paper we shall use three basic methods. First, we shall prepare symbolic calculus for non-isotropic pseudodifferential operators of $(\varrho, \delta)$-type in Section 3. This will be applied for Grushin operators in Section 4. Second, method of bracket calculus given in [2] will be used in Section 5. Third, method of FBI-transformation given in [5] and [6] will be developed slightly and used in Section 6 to complete the proof of our main result Theorem 2.1. It looks that both methods of Sections 5 and 6 are interesting although the result of Section 
6 includes that of Section 5. Thus the original problem is almost completely solved for Grushin operators in this paper, while an interesting and challenging problem occurs, (see Remark 2.2).

\section{§2. Main Results}

We denote $x=\left(x_{1}, x_{2}, \ldots, x_{n}\right) \in \mathbf{R}^{n}$, and $D=\left(D_{1}, D_{2}, \ldots, D_{n}\right), D_{j}=$ $-i \partial_{x_{j}}, j=1,2, \ldots, n$ as usual.

First we remember the definition of Gevrey functions.

Definition 2.1. Let $\Omega$ be an open set in $\mathbf{R}^{n}$ and $\varphi \in C^{\infty}(\Omega)$. Then we say that $\varphi \in G^{\{\theta\}}(\Omega), \theta>0$, if for any compact subset $K$ of $\Omega$ there are positive constants $C_{0}$ and $C_{1}$ such that

$$
\sup _{x \in K}\left|D^{\alpha} \varphi(x)\right| \leq C_{0} C_{1}^{|\alpha|} \alpha !^{\theta}, \quad \alpha \in \mathbf{Z}_{+}^{n} .
$$

We say that $\varphi \in G^{\left\{d_{1}, d_{2}, \ldots, d_{n}\right\}}(\Omega), 0<d_{1}, d_{2}, \ldots, d_{n}<\infty$, if for any compact subset $K$ of $\Omega$ there are positive constants $C_{0}$ and $C_{1}$ such that

$$
\sup _{x \in K}\left|D^{\alpha} \varphi(x)\right| \leq C_{0} C_{1}^{|\alpha|} \alpha_{1} !^{d_{1}} \alpha_{2} !^{d_{2}} \cdots \alpha_{n} !^{d_{n}}, \quad \alpha \in \mathbf{Z}_{+}^{n} .
$$

Proposition 2.1. Let $\varphi \in C^{\infty}(\Omega)$. If for any compact subset $K$ of $\Omega$ there are positive constants $C_{0}$ and $C_{1}$ such that

$$
\sup _{x \in K}\left|D_{j}^{k} \varphi\right| \leq C_{0} C_{1}^{k} k !^{d_{j}}, \quad j=1,2, \ldots, n, \quad k \in \mathbf{Z}_{+} .
$$

Then we have $\varphi \in G^{\left\{d_{1}, d_{2}, \ldots, d_{n}\right\}}(\Omega)$.

Next we remember the Grushin operators in a general form. We write $(x, y)=\left(x_{1}, \ldots, x_{k}, y_{1}, \ldots, y_{n}\right) \in \mathbf{R}^{k+n}=\mathbf{R}^{N}$. Let $m$ be an even positive integer and let $\sigma=\left(\sigma_{1}, \sigma_{2}, \ldots, \sigma_{k}\right), q=\left(q_{1}, q_{2}, \ldots, q_{k}\right)$ whose elements are rational numbers such that

$$
\begin{gathered}
\sigma_{1}, \ldots, \sigma_{p}>0, \quad \sigma_{p+1}=\cdots=\sigma_{k}=0, \quad(0 \leq p \leq k) \\
q_{1} \geq q_{2} \geq \cdots \geq q_{k} \geq 0, \quad q_{1}>0 .
\end{gathered}
$$

Furthermore, we assume

$$
\begin{aligned}
m q_{j} & \in \mathbf{Z}, \quad j=1, \ldots, k \\
\frac{m q_{j}}{\sigma_{j}} \in \mathbf{Z}, & j=1, \ldots, p,
\end{aligned}
$$


and

$$
1+q_{k}>\sigma_{0}=\max \left(\sigma_{1}, \sigma_{2}, \ldots, \sigma_{p}\right) .
$$

We divide $x$ into two parts such as $x=\left(x^{\prime}, x^{\prime \prime}\right)$ when $1 \leq p<k$, where $x^{\prime}=\left(x_{1}, \ldots, x_{p}\right)$ and $x^{\prime \prime}=\left(x_{p+1}, \ldots, x_{k}\right)$. We consider $x=x^{\prime}$ when $p=k$ and $x=x^{\prime \prime}, p=0$ when $\sigma=(0, \ldots, 0)$.

Now we consider the differential operator with polynomial coefficients:

$$
\begin{aligned}
P\left(x^{\prime}, y, D_{x}, D_{y}\right)= & \sum_{\substack{\langle\sigma, \nu\rangle+|\gamma|=\langle q, \alpha\rangle+|\alpha+\beta|-m \\
|\alpha+\beta| \leq m}} a_{\alpha \beta \nu \gamma} x^{\prime \nu} y^{\gamma} D_{x}^{\alpha} D_{y}^{\beta}, \\
& a_{\alpha \beta \nu \gamma} \in \mathbf{C}, \quad \alpha, \nu \in \mathbf{Z}_{+}^{k}, \quad \beta, \gamma \in \mathbf{Z}_{+}^{n},
\end{aligned}
$$

where $a_{\alpha \beta \nu \gamma}$ can be non-zero only when $|\gamma|=\langle q, \alpha\rangle+|\alpha+\beta|-m-\langle\sigma, \nu\rangle$ is a non-negative integer and we write $|\alpha+\beta|=|\alpha|+|\beta|$. We may consider $\nu=\left(\nu_{1}, \nu_{2}, \ldots, \nu_{p}, 0, \ldots, 0\right)$.

We can see that the symbol $P\left(x^{\prime}, y, \xi, \eta\right)$ satisfies the following condition.

Condition 1 (quasi-homogeneity). We have

$P\left(\lambda^{-\sigma} x^{\prime}, \lambda^{-1} y, \lambda^{1+q} \xi, \lambda \eta\right)=\lambda^{m} P\left(x^{\prime}, y, \xi, \eta\right), \quad \lambda>0, \quad y, \eta \in \mathbf{R}^{n}, \quad x, \xi \in \mathbf{R}^{k}$, where $\lambda^{-\sigma} x^{\prime}=\left(\lambda^{-\sigma_{1}} x_{1}, \ldots, \lambda^{-\sigma_{p}} x_{p}\right)$ and $\lambda^{1+q} \xi=\left(\lambda^{1+q_{1}} \xi_{1}, \ldots, \lambda^{1+q_{k}} \xi_{k}\right)$.

We add the following two conditions on $P$.

Condition 2 (ellipticity). The operator $P$ is elliptic for $\left|x^{\prime}\right|+|y|=1$.

Condition 3 (non-zero eigenvalue). For all $\omega \in \mathbf{R}^{k},|\omega|=1$, the equation

$$
P\left(x^{\prime}, y, \omega, D_{y}\right) v(y)=0 \quad \text { in } \quad \mathbf{R}^{n}
$$

has no non-trivial solution in $\mathcal{S}\left(\mathbf{R}_{y}^{n}\right)$.

We denote $\varrho_{0}=\left(1+q_{k}\right) /\left(1+q_{p}\right) \leq 1, \sigma_{0}=\max \left(\sigma_{1}, \ldots, \sigma_{p}\right)<1+q_{k}$ by assumption and $\delta=\sigma_{0} /\left(1+q_{k}\right)<1$. We set the Gevrey index $\theta_{j}=$ $\max \left(\left(1+q_{j}\right) /\left(1+q_{k}\right), 1 /\left(1-\varrho_{0} \delta\right)\right), \delta=\sigma_{0} /\left(1+q_{k}\right)$, for $j=1,2, \ldots, p$ and $\theta_{j}=\left(1+q_{j}\right) /\left(1+q_{k}\right)$ for $j=p+1, \ldots, k$. We set $d=\left(\theta_{1}+q_{1}\right) /\left(1+q_{1}\right) \cdot I_{n}=$ $\left(\left(\theta_{1}+q_{1}\right) /\left(1+q_{1}\right), \ldots,\left(\theta_{1}+q_{1}\right) /\left(1+q_{1}\right)\right)$.

We shall prove the following theorem in Sections 4 through 6 under these conditions on $P$. 
Theorem 2.1 (cf. [26]). Let $\Omega$ be an open neighborhood of $(0,0) \in \mathbf{R}^{k+n}$ and consider the equation

$$
P\left(x^{\prime}, y, D_{x}, D_{y}\right) u(x, y)=f(x, y) \quad \text { in } \quad \Omega,
$$

where $u(x, y) \in C^{\infty}(\Omega)$ and $f(x, y) \in G_{x, y}^{\{\theta, d\}}(\Omega)$. Then we have $u \in G_{x, y}^{\{\theta, d\}}(\Omega)$. Here $\theta=\left(\theta_{1}, \theta_{2}, \ldots, \theta_{k}\right)$ and $d=\left(\theta_{1}+q_{1}\right) /\left(1+q_{1}\right) \cdot I_{n}$ as given above.

Remark 2.1. In the above theorem we can see that

(i) $\theta_{1}=1 \Longleftrightarrow(\theta, d)=(1, \ldots, 1)$,

(ii) $\theta_{1}>1 \Longleftrightarrow 1<d<\theta_{1}$.

Remark 2.2. Beyond the Conditions 1 through 3, the major hypothesis

$$
1+q_{k}>\sigma_{0}=\max \left(\sigma_{1}, \ldots, \sigma_{p}\right)
$$

plays an essential role throughout the paper. Hence, a problem to weaken this hypothesis remains open.

Examples 1. (a) For the operator $L=\partial_{y}^{2}+\left(x^{2 l}+y^{2 k}\right) \partial_{x}^{2},(l, k=$ $1,2, \ldots)$, given in the introduction, we have $q_{1}=k, \sigma_{1}=k / l, \delta=k /(l(1+k))$ and $\theta=1 /(1-\delta)=l(1+k) /(l(1+k)-k), d=(\theta+k) /(1+k)$. The optimality of the index $\{\theta, d\}$ was shown in the paper [28].

(b) For the operator $M=\partial_{y}^{2}+\left(x^{2 l}+y^{2 k}\right)\left(\partial_{x}^{2}+\partial_{z}^{2}\right),(l, k=1,2, \ldots)$, given in the introduction, we have $q_{1}=q_{2}=k, \sigma_{1}=k / l, \sigma_{2}=0, x^{\prime}=x_{1}, x^{\prime \prime}=$ $x_{2}, \delta=k /(l(1+k)), \theta_{1}=1 /(1-\delta)=(l(1+k)) /(l(1+k)-k), \theta_{2}=1$ and $d=\left(\theta_{1}+k\right) /(1+k)$.

(c) Let $q_{1}, q_{2}, \ldots, q_{k}$ be integers such that

$$
q_{1} \geq q_{2} \geq \cdots \geq q_{k} \geq 0, \quad q_{1}>q_{k} .
$$

Then the operator

$$
D_{y}^{2}+\sum_{j=1}^{k} y^{2 q_{j}} D_{x_{j}}^{2}
$$

has $G_{x, y}^{\left\{\theta,\left(\theta_{1}+q_{1}\right) /\left(1+q_{1}\right)\right\}}$-hypoellipticity in a neighborhood of the origin in $\mathbf{R}_{x, y}^{k+1}$, where $\theta=\left(\left(\left(1+q_{1}\right) /\left(1+q_{k}\right)\right),\left(\left(1+q_{2}\right) /\left(1+q_{k}\right)\right), \ldots, 1\right)$. Note that $\delta=0$ in this case. 
We shall show now the optimality of the exponent $\left\{\theta,\left(\left(\theta_{1}+q_{1}\right) /\left(1+q_{1}\right)\right)\right\}$ at the origin by the method given in [2]. By the results of [32], we know that there exists a positive number $a$ such that the ordinary differential equation

$$
v^{\prime \prime}(t)-\sum_{j=1}^{k-1} t^{2 q_{j}} v(t)+a^{2} t^{2 q_{k}} v(t)=0
$$

has a non-trivial solution $v(t) \in L_{2}(\mathbf{R})$. Then by [26], we know $v(t) \in$ $\mathcal{S}_{1 /\left(1+q_{1}\right)}^{q_{1} /\left(1+q_{1}\right)}(\mathbf{R})$ which is a space of Gel'fand-Shilov, [8]. That is to say, there are positive constants $C_{0}$ and $C_{1}$ such that

$$
\sup _{-\infty<t<\infty}\left|t^{l} \partial_{t}^{j} v(t)\right| \leq C_{0} C_{1}^{l+j} l !^{\frac{1}{1+q_{1}}} j^{\frac{q_{1}}{1+q_{1}}}, \quad l, j=1,2, \ldots
$$

Then by [8], we can see that for any small positive number $\varepsilon$, there are infinitely many numbers, $j_{i}, i=1,2, \ldots$, such that

$$
\left|\partial_{t}^{j_{i}} v(0)\right| \geq \varepsilon^{j_{i}} j_{i} !^{\frac{q_{1}}{1+q_{1}}-\varepsilon} .
$$

Now we define the function

$$
\begin{aligned}
u(x, y)= & \int_{0}^{\infty} \exp \left[i\left(x_{1} \varrho^{\frac{1+q_{1}}{1+q_{k}}}+x_{2} \varrho^{\frac{1+q_{2}}{1+q_{k}}}+\cdots+x_{k-1} \varrho^{\frac{1+q_{k-1}}{1+q_{k}}}\right)+a \varrho x_{k}\right] \\
& \times v\left(\varrho^{\frac{1}{1+q_{k}}} y\right) e^{-\varrho} d \varrho .
\end{aligned}
$$

We can see the function $u$ in (2.10) is a solution of the differential equation

$$
\left(D_{y}^{2}+\sum_{j=1}^{k} y^{2 q_{j}} D_{x_{j}}^{2}\right) u(x, y)=0
$$

in a neighborhood of the origin. Furthermore, we can easily see that

$$
\left|\partial_{x_{j}}^{l} u(0,0)\right| \sim l !^{\frac{1+q_{j}}{1+q_{k}}}, \quad j=1, \ldots, k
$$

and

$$
\left|\partial_{y}^{l} u(0,0)\right| \sim\left|v^{(l)}(0)\right| \int_{0}^{\infty} \varrho^{\frac{l}{1+q_{k}}} e^{-\varrho} d \varrho .
$$

By (2.8) and (2.9) we have

$$
\left|\partial_{y}^{l} u(0,0)\right| \sim l !^{\frac{q_{1}}{1+q_{1}}} l !^{\frac{1}{1+q_{k}}} .
$$


We see

$$
\frac{q_{1}}{1+q_{1}}+\frac{1}{1+q_{k}}=\frac{q_{1}+\frac{1+q_{1}}{1+q_{k}}}{1+q_{1}}=\frac{\theta_{1}+q_{1}}{1+q_{1}} .
$$

This shows the optimality of the exponent $\left\{\theta,\left(\left(\theta_{1}+q_{1}\right) /\left(1+q_{1}\right)\right)\right\}$ for the operator given in (2.6).

\section{§3. Some Elementary Preparation for Non-isotropic Pseudodifferential Operators of $(\varrho, \delta)$-type}

We write $x=\left(x_{1}, x_{2}, \ldots, x_{n}\right)$ and $\xi=\left(\xi_{1}, \xi_{2}, \ldots, \xi_{n}\right) \in \mathbf{R}^{n}$. Let $\varrho=$ $\left(\varrho_{1}, \varrho_{2}, \ldots, \varrho_{n}\right), 0<\varrho_{j} \leq 1, j=1,2, \ldots, n$ and $0 \leq \delta<1$. We set

$$
|\xi|_{\varrho}=\left|\xi_{1}\right|^{\varrho_{1}}+\left|\xi_{2}\right|^{\varrho_{2}}+\cdots+\left|\xi_{n}\right|^{\varrho_{n}} .
$$

Let $\Omega \subset \mathbf{R}^{n}$ be an open set. We divide $x \in \Omega$ such as

$$
x=\left(x_{1}, x_{2}, \ldots, x_{p}, x_{p+1}, \ldots, x_{n}\right)=\left(x^{\prime}, x^{\prime \prime}\right) .
$$

We consider $x=x^{\prime}$ when $p=n$ and $x=x^{\prime \prime}$ when $p=0$. We divide also the multi-index $\alpha=\left(\alpha^{\prime}, \alpha^{\prime \prime}\right)=\left(\alpha_{1}, \ldots, \alpha_{p}, \alpha_{p+1}, \ldots, \alpha_{n}\right), 1 \leq p \leq n$.

Definition 3.1. A function $a(x, \xi) \in C^{\infty}\left(\Omega \times \mathbf{R}^{n}\right)$ is said in the symbol class $S_{\varrho, \delta}^{m}\left(\Omega \times \mathbf{R}^{n}\right)$, if for any compact subset $K$ of $\Omega$ there are positive constants $C_{0}, C_{1}$ and $B$ such that

$$
\begin{aligned}
& \sup _{x \in K}\left|a_{(\beta)}^{(\alpha)}(x, \xi)\right| \leq C_{0} C_{1}^{|\alpha+\beta|} \alpha ! \beta !\left(1+|\xi|_{\varrho}\right)^{m-|\alpha|+\delta\left|\beta^{\prime}\right|}, \quad|\xi|_{\varrho} \geq B \\
& \sup _{x \in K}\left|a_{(\beta)}(x, \xi)\right| \leq C_{0} C_{1}^{|\beta|} \beta !, \quad|\xi|_{\varrho} \leq B .
\end{aligned}
$$

Here we use the notation

$$
a_{(\beta)}^{(\alpha)}(x, \xi)=\partial_{\xi}^{\alpha} D_{x}^{\beta} a(x, \xi), \quad \alpha, \beta \in \mathbf{Z}_{+}^{n} .
$$

For $u(x) \in C_{0}^{\infty}(\Omega)$ the pseudodifferential operator $a(x, D)$ is defined by the formula

$$
\begin{aligned}
a(x, D) u(x) & =(2 \pi)^{-n} \iint e^{i\langle x-y, \xi\rangle} a(x, \xi) u(y) d y d \xi \\
& =(2 \pi)^{-n} \int e^{i\langle x, \xi\rangle} a(x, \xi) \hat{u}(\xi) d \xi \\
& =\int K(x, y) u(y) d y
\end{aligned}
$$


where the Schwartz kernel $K(x, y) \in \mathcal{D}^{\prime}\left(\Omega \times \mathbf{R}^{n}\right)$ is an oscillatory integral defined by the formula

$$
K(x, y)=(2 \pi)^{-n} \int e^{i\langle x-y, \xi\rangle} a(x, \xi) d \xi .
$$

Theorem 3.1. We set $\varrho_{0}=\max \left(\varrho_{1}, \ldots, \varrho_{p}\right)$. Then we have

$$
K(x, y) \in G_{x, y}^{\left\{\theta, \frac{1}{\varrho}\right\}}\left(\Omega \times \mathbf{R}^{n} \backslash \Delta\right)
$$

where $\Delta=\{(x, x) ; x \in \Omega\}$ and

$$
\begin{aligned}
\theta & =\left(\theta_{1}, \ldots, \theta_{n}, \theta_{1}, \ldots, \theta_{n}\right), \quad \frac{1}{\varrho}=\left(\frac{1}{\varrho_{1}}, \ldots, \frac{1}{\varrho_{n}}\right), \\
\theta_{j} & =\max \left(\frac{1}{\varrho_{j}}, \frac{1}{1-\varrho_{0} \delta}\right), \quad j=1, \ldots, p ; \quad \theta_{j}=\frac{1}{\varrho_{j}}, \quad j=p+1, \ldots, n .
\end{aligned}
$$

Proof. The idea for the proof is similar to that used in the paper [22] and in the lecture notes [27], so we shall mention briefly the essential parts of the proof.

Let $U$ be any compact subset of $\Omega \times \mathbf{R}^{n} \backslash \Delta$. First we shall estimate the $y$-derivatives of $K(x, y)$ on $U$. For every $\beta \in \mathbf{Z}_{+}^{n}$ we have in the oscillatory sense:

$$
D_{y}^{\beta} K(x, y)=(2 \pi)^{-n} \int e^{i\langle x-y, \xi\rangle}(-\xi)^{\beta} a(x, \xi) d \xi .
$$

We denote $r=d / n, d=\operatorname{dis}(U, \Delta)$. For any $(x, y) \in U$ we can find some $l, 1 \leq l \leq n$, such that $\left|x_{l}-y_{l}\right| \geq r$. Let us write

$$
\begin{aligned}
\left\langle\frac{1}{\varrho}, \beta\right\rangle & =\frac{1}{\varrho_{1}} \beta_{1}+\frac{1}{\varrho_{2}} \beta_{2}+\cdots+\frac{1}{\varrho_{n}} \beta_{n}, \\
N & =\left[\left\langle\frac{1}{\varrho}, \beta\right\rangle\right]+1 .
\end{aligned}
$$

Here we denote by $[a]$ the largest integer smaller than or equal to $a$.

Then we have

$$
\begin{aligned}
D_{y}^{\beta} K(x, y)= & (2 \pi)^{-n} \int e^{i\langle x-y, \xi\rangle}(-\xi)^{\beta} a(x, \xi) d \xi \\
= & (2 \pi)^{-n} \int_{|\xi|_{\rho} \leq B} e^{i\langle x-y, \xi\rangle}\left((-\xi)^{\beta} a(x, \xi)\right) d \xi \\
& +(2 \pi)^{-n} \int_{|\xi|_{\rho} \geq B} e^{i\langle x-y, \xi\rangle}\left((-\xi)^{\beta} a(x, \xi)\right) d \xi \\
\equiv & I_{1}+I_{2} .
\end{aligned}
$$


We assume $m<0$ and $|m|$ sufficiently large for simplicity. By the assumption (3.2), we have the estimate of the form

$$
\left|I_{1}\right| \leq M B^{|\beta|} .
$$

For $I_{2}$ we have

$$
\begin{aligned}
I_{2} & =\left(x_{l}-y_{l}\right)^{-N} \int_{|\xi|_{\varrho} \geq B} D_{\xi_{l}}^{N} e^{i\langle x-y, \xi\rangle}(-\xi)^{\beta} a(x, \xi) d \xi \\
& =\text { Boundary terms }+\int_{|\xi|_{\varrho} \geq B} e^{i\langle x-y, \xi\rangle} D_{\xi_{l}}^{N}(-\xi)^{\beta} a(x, \xi) d \xi .
\end{aligned}
$$

For the boundary terms we have the same type of the estimates as for $I_{1}$. By the assumption $(3.1)$ on the symbol $a(x, \xi)$ the integrand of the last term is estimated by

$$
\begin{aligned}
& \left|\sum_{k=0}^{\beta_{l}} \frac{N ! \beta_{l} !}{k !(N-k) !\left(\beta_{l}-k\right) !} \xi^{\beta} \xi_{l}^{-k} D_{\xi_{l}}^{N-k} a(x, \xi)\right| \\
& \quad \leq C_{0} C_{1}^{N} N ! \sum_{k=0}^{\beta_{l}}\left(\begin{array}{c}
\beta_{l} \\
k
\end{array}\right)\left|\xi_{1}\right|^{\beta_{1}}\left|\xi_{2}\right|^{\beta_{2}} \cdots\left|\xi_{l}\right|^{\beta_{l}-k} \cdots\left|\xi_{n}\right|^{\beta_{n}}\left(1+|\xi|_{\varrho}\right)^{-(N-k)+m} \\
& \quad \leq C_{0}^{\prime} C_{1}^{\prime|\beta|} \beta !^{\theta}\left(1+|\xi|_{\varrho}\right)^{m}, \quad|\xi|_{\varrho} \geq B .
\end{aligned}
$$

Next we shall estimate $x$-derivatives of $K(x, y)$ on $U$. We have

$$
\begin{aligned}
D_{x}^{\alpha} K(x, y) & =(2 \pi)^{-n} \sum_{\gamma+\tau=\alpha}\left(\begin{array}{l}
\alpha \\
\gamma
\end{array}\right) \int e^{i\langle x-y, \xi\rangle} \xi^{\gamma} a_{(\tau)}(x, \xi) d \xi \\
& =(2 \pi)^{-n} \sum_{\gamma+\tau=\alpha}\left(-\left(x_{l}-y_{l}\right)\right)^{-N(\tau)} \int e^{i\langle x-y, \xi\rangle} D_{\xi_{l}}^{N(\tau)}\left(\xi^{\gamma} a_{(\tau)}(x, \xi)\right) d \xi
\end{aligned}
$$

where

$$
N(\tau)=\left[\left\langle\frac{1}{\varrho}, \gamma\right\rangle+\delta\left|\tau^{\prime}\right|\right]+1 .
$$

As we can see in the proof for the isotropic case given in [22] and [27], principally we need to estimate the integrand of the last member for $|\xi|_{\varrho} \geq B \cdot N$. By using the assumption (3.1), we get the estimate of the form

$$
\left|D_{\xi_{l}}^{N(\tau)}\left(\xi^{\gamma} a_{(\tau)}(x, \xi)\right)\right| \leq C_{0} C_{1}^{|\alpha|} \gamma !^{\frac{1}{\varrho}} \tau^{\prime} !^{1+\delta} \tau^{\prime \prime} !\left(1+|\xi|_{\varrho}\right)^{m} .
$$

If we assume the fact

$$
1+\delta \leq \theta_{j}, \quad(j=1,2, \ldots, p)
$$


then the left-hand side of the above inequality is estimated by the quantity

$$
C_{0}^{\prime} C_{1}^{\prime|\alpha|} \gamma !^{!^{\frac{1}{\varrho}}} \tau !^{\theta} \leq C_{0}^{\prime} C_{1}^{\prime|\alpha|} \alpha !^{\theta}
$$

where the constants $C_{0}^{\prime}$ and $C_{1}^{\prime}$ are taken independent of $\tau$ and $\alpha$.

It remains to prove the inequality (3.5).

(1) The case where $1 /\left(1-\varrho_{0} \delta\right) \leq 1 / \varrho_{j}=\theta_{j}, j=1,2, \ldots, p$.

Take $\varrho_{j}=\varrho_{0}=\max \left(\varrho_{1}, \ldots, \varrho_{p}\right)$, then we have the inequality

$$
\frac{1}{1-\varrho_{0} \delta} \leq \frac{1}{\varrho_{0}}
$$

from which we get the inequality

$$
1+\delta \leq \frac{1}{\varrho_{0}} \leq \frac{1}{\varrho_{j}}=\theta_{j}, \quad j=1,2, \ldots, p
$$

(2) The case where there is some number $j, 1 \leq j \leq p$, such that

$$
\frac{1}{\varrho_{j}}<\frac{1}{1-\varrho_{0} \delta}=\theta_{j} .
$$

Then we have $1-\varrho_{0} \delta<\varrho_{j}$, from which we have

$$
1<\varrho_{j}+\varrho_{0} \delta \leq \varrho_{0}(1+\delta) .
$$

On the other hand, we have the equivalence relation

$$
1+\delta \leq \frac{1}{1-\varrho_{0} \delta}\left(\leq \theta_{j}\right) \Longleftrightarrow 1 \leq \varrho_{0}(1+\delta),
$$

which fits the above inequality and we have (3.5).

Next we shall consider the pseudolocal property of $a(x, D)$.

Lemma 3.1. Let $K$ be a compact subset of an open set $V \subset \mathbf{R}^{n}$. Then there is a sequence of functions $\left\{g_{l}(x)\right\} \subset C_{0}^{\infty}(V)$ and a constant $C$ such that

$$
\begin{gathered}
\left|D^{\alpha} g_{l}(x)\right| \leq C^{l} \alpha !, \quad|\alpha| \leq l, \quad l=0,1,2, \ldots, \\
g_{l}(x)=1, \quad x \in K, \quad l=0,1,2, \ldots
\end{gathered}
$$

Lemma 3.2. Let $\Omega^{\prime} \subset \overline{\Omega^{\prime}} \subset \subset \Omega$. Then for $f(x) \in C_{0}^{\infty}\left(\Omega^{\prime}\right)$ we have the estimate of the form

$$
\begin{aligned}
\left|D_{x}^{\alpha} a(x, D) f(x)\right| \leq & \sum_{\gamma+\tau=\alpha}\left(\begin{array}{l}
\alpha \\
\gamma
\end{array}\right) C^{1+|\tau|} \tau ! \operatorname{Vol}\left(\Omega^{\prime}\right) \\
& \cdot \sup _{x \in \Omega^{\prime}}\left|D_{x}^{\gamma}(1+|D| \varrho)^{\delta\left|\tau^{\prime}\right|} f(x)\right|,
\end{aligned}
$$


where the constant $C$ is taken independent of $\alpha$ and $\tau$ and the precise meaning of $\left(1+|D|_{\varrho}\right)^{\delta\left|\tau^{\prime}\right|}$ will be given in the proof.

Proof of Lemma 3.2. We have

$$
\begin{aligned}
D_{x}^{\alpha}\{a(x, D) f(x)\} & =(2 \pi)^{-n} \sum_{\gamma+\tau=\alpha}\left(\begin{array}{l}
\alpha \\
\gamma
\end{array}\right) \iint D_{x}^{\gamma} e^{i\langle x-y, \xi\rangle} a_{(\tau)}(x, \xi) f(y) d y d \xi \\
& =(2 \pi)^{-n} \sum_{\gamma+\tau=\alpha}\left(\begin{array}{l}
\alpha \\
\gamma
\end{array}\right) \iint\left(-D_{y}\right)^{\gamma} e^{i\langle x-y, \xi\rangle} a_{(\tau)}(x, \xi) f(y) d y d \xi \\
& =(2 \pi)^{-n} \sum_{\gamma+\tau=\alpha}\left(\begin{array}{l}
\alpha \\
\gamma
\end{array}\right) \iint e^{i\langle x-y, \xi\rangle} a_{(\tau)}(x, \xi) D_{y}^{\gamma} f(y) d y d \xi
\end{aligned}
$$

We know that

$$
\left|a_{(\tau)}(x, \xi)\right| \leq C_{0} C_{1}^{|\tau|} \tau !\left(1+|\xi|_{\varrho}\right)^{\delta\left|\tau^{\prime}\right|+m} .
$$

We assume $m<0$ and $|m|$ sufficiently large for simplicity. We note that

$$
\begin{aligned}
\left(1+|\xi|_{\varrho}\right)^{\delta\left|\tau^{\prime}\right|} & =\left(1+\left|\xi_{1}\right|^{\varrho_{1}}+\cdots+\left|\xi_{n}\right|^{\varrho_{n}}\right)^{\delta\left|\tau^{\prime}\right|} \\
& \leq C^{\delta\left|\tau^{\prime}\right|}\left(1+\xi_{1}^{2\left[\frac{\underline{\varrho}_{1} \delta\left|\tau^{\prime}\right|}{2}+1\right]}+\cdots+\xi_{n}^{2\left[\frac{\underline{\rho}_{n} \delta\left|\tau^{\prime}\right|}{2}+1\right]}\right)
\end{aligned}
$$

where the constant $C$ is taken independent of $\tau$. We rewrite the last member of the above equality as follows:

$$
\begin{aligned}
& =(2 \pi)^{-n} \sum_{\gamma+\tau=\alpha}\left(\begin{array}{l}
\alpha \\
\gamma
\end{array}\right) \iint\left(1-D_{y_{1}}^{2\left[\frac{\underline{\rho}_{1} \delta\left|\tau^{\prime}\right|}{2}+1\right]}-\cdots-D_{y_{n}}^{2\left[\frac{\underline{e_{n} \delta\left|\tau^{\prime}\right|}}{2}+1\right]}\right) e^{i\langle x-y, \xi\rangle} . \\
& \cdot\left(1+\xi_{1}^{2\left[\frac{\underline{e}_{1} \delta\left|\tau^{\prime}\right|}{2}+1\right]}+\cdots+\xi_{n}^{2\left[\frac{e_{n} \delta\left|\tau^{\prime}\right|}{2}+1\right]}\right)^{-1} a_{(\tau)}(x, \xi) D_{y}^{\gamma} f(y) d y d \xi \\
& =(2 \pi)^{-n} \sum_{\gamma+\tau=\alpha}\left(\begin{array}{l}
\alpha \\
\gamma
\end{array}\right) \iint e^{i\langle x-y, \xi\rangle}\left(1+\xi_{1}^{2\left[\frac{\underline{\rho}_{1} \delta\left|\tau^{\prime}\right|}{2}+1\right]}+\cdots+\xi_{n}^{2\left[\frac{\underline{e}_{n} \delta\left|\tau^{\prime}\right|}{2}+1\right]}\right)^{-1} \\
& \cdot a_{(\tau)}(x, \xi)\left(1-D_{y_{1}}^{2\left[\frac{\underline{e}_{1} \delta\left|\tau^{\prime}\right|}{2}+1\right]}-\cdots-D_{y_{n}}^{2\left[\frac{\underline{o}_{n} \delta\left|\tau^{\prime}\right|}{2}+1\right]}\right) D_{y}^{\gamma} f(y) d y d \xi
\end{aligned}
$$

This is the precise meaning of $\left(1+|D|_{\varrho}\right)^{\delta\left|\tau^{\prime}\right|}$ and from where we have (3.8).

Theorem 3.2. $\quad$ Let $a(x, D)$ be as above. Then we have the assertion:

(3.9) $u \in \mathcal{E}^{\prime}(\Omega) \cap G^{\{\theta\}}(\omega) \Rightarrow a(x, D) u \in \mathcal{D}^{\prime}(\Omega) \cap G^{\{\theta\}}(\omega), \quad(\omega \subset \Omega)$, $\theta_{j}=\max \left(\frac{1}{\varrho_{j}}, \frac{1}{1-\varrho_{0} \delta}\right), \quad j=1,2, \ldots, p ; \quad \theta_{j}=\frac{1}{\varrho_{j}}, \quad j=p+1, \ldots, n$. 
Proof. Let $U$ be a bounded open set and $\bar{U} \subset \omega$. Then there is a positive number $d$ such that $d i s\left(U, \omega^{c}\right)>d>0$. By virtue of Lemma 3.1, we can take a sequence of functions $g_{l}(x) \in C_{0}^{\infty}(\omega)$ such that $g_{l}(x)=1$ for $x \in\{x ; \operatorname{dis}(x, U)<$ $d / 4\}$ and

$$
\left|D^{\alpha} g_{l}(x)\right| \leq C^{l} \alpha !, \quad|\alpha| \leq l, \quad l=0,1,2, \ldots .
$$

Now take $l=2|\alpha|$. Then we have for $x \in U$

$$
\begin{aligned}
D^{\alpha}\{a(x, D) u(x)\}= & D^{\alpha}\left\{a(x, D) g_{l} u(x)\right\} \\
& +D^{\alpha} \int K(x, y)\left\{1-g_{l}(y)\right\} u(y) d y .
\end{aligned}
$$

By using (3.8), we have

$$
\begin{aligned}
& \left|D^{\alpha}\left\{a(x, D) g_{l} u(x)\right\}\right| \leq \sum_{\gamma+\tau=\alpha}\left(\begin{array}{l}
\alpha \\
\gamma
\end{array}\right) C^{|\tau|+1} \tau ! \sup _{x \in \omega}\left|D^{\gamma}\left(1+|D|_{\varrho}\right)^{\delta\left|\tau^{\prime}\right|}\left\{g_{l}(x) u(x)\right\}\right| \\
& \leq \tilde{C}_{1}{ }^{|\alpha|+1} \sum_{\gamma+\tau=\alpha} \tilde{C}_{2}^{|\gamma|+1} \gamma !^{\theta} \tau !\left(\left|\tau^{\prime}\right| !^{\varrho_{1} \delta \theta_{1}}+\cdots+\left|\tau^{\prime}\right| !^{\varrho_{n} \delta \theta_{n}}\right) \\
& \leq C_{0}^{\prime} C_{1}^{\prime|\alpha|} \gamma !^{\theta} \tau^{\prime \prime} ! \sum_{j=1}^{n}\left|\tau^{\prime}\right| !^{1+\varrho_{j} \delta \theta_{j}} \\
& \leq C_{0} C_{1}^{|\alpha|} \gamma !^{\theta} \tau !^{\theta} \leq C_{0} C_{1}^{|\alpha|} \alpha !^{\theta},
\end{aligned}
$$

where the constants $C_{0}$ and $C_{1}$ are taken independent of $\alpha$. Here we need to estimate the last summation in the above inequalities. We shall show that we have

$$
\begin{aligned}
& 1+\varrho_{k} \delta \theta_{k} \leq \theta_{j}, \quad(k, j=1,2, \ldots, p) \\
& 1+\varrho_{k} \delta \theta_{k}=1+\delta \leq \theta_{j}, \quad(k=p+1, \ldots, n ; j=1, \ldots, p),
\end{aligned}
$$

from where the last inequalities are derived completely.

(1) The case $\theta_{k}=1 / \varrho_{k} \geq 1 /\left(1-\varrho_{0} \delta\right),(k=1, \ldots, p)$. In this case, we have

$$
1-\varrho_{0} \delta \geq \varrho_{k}, \quad k=1, \ldots, p .
$$

Taking $\varrho_{k}=\varrho_{0}$, we have

$$
\frac{1}{\varrho_{0}} \geq 1+\delta
$$

and

$$
\theta_{j}=\frac{1}{\varrho_{j}} \geq \frac{1}{\varrho_{0}}, \quad j=1, \ldots, p .
$$


(2) The case there is a number $k, 1 \leq k \leq p$, such that $1 / \varrho_{k}<1 /\left(1-\varrho_{0} \delta\right)=\theta_{k}$. In this case, we have

$$
1-\varrho_{0} \delta<\varrho_{k}
$$

from where we have

$$
1<\varrho_{0}(1+\delta)
$$

On the other hand, we have

$$
1+\varrho_{0} \delta \theta_{k}=1+\varrho_{0} \delta \frac{1}{1-\varrho_{0} \delta}=\frac{1}{1-\varrho_{0} \delta} \leq \theta_{j}, \quad j=1, \ldots, p .
$$

If there is another number $k, 1 \leq k \leq p$, such that $\theta_{k}=1 / \varrho_{k} \leq 1 /\left(1-\varrho_{0} \delta\right)$, by applying (3.10) we have

$$
1+\varrho_{k} \delta \theta_{k}=1+\delta \leq \frac{1}{1-\varrho_{0} \delta} \leq \theta_{j}, \quad j=1, \ldots, p .
$$

In what follows we shall consider the symbolic calculus and the Gevrey hypoellipticity of the pseudodifferential operators with symbols given in Definition 3.1. The method is similar to that of [22], [27, Section 12] with some revision just like above. Therefore we omit the proof. Let $\Omega^{\prime}$ be a relatively compact open subset of $\Omega$. Let $a(x, \xi) \in S_{\varrho, \delta}^{m^{\prime}}\left(\Omega \times \mathbf{R}^{n}\right), b(x, \xi) \in S_{\varrho, \delta}^{m^{\prime \prime}}\left(\Omega \times \mathbf{R}^{n}\right)$. Now consider the product

$$
r(x, D) \equiv a(x, D) h(x) b(x, D),
$$

where $h(x) \in C_{0}^{\infty}(\Omega)$ such that $h(x) \equiv 1$ in a neighborhood of $\overline{\Omega^{\prime}}$. The symbol $r(x, \xi)$ of $r(x, D)$ is given by

$$
\begin{aligned}
r(x, \xi) & =a(x, D+\xi) h(x) b(x, \xi) \\
& =(2 \pi)^{-n} \int e^{i\left\langle x-y, \xi^{\prime}\right\rangle} a\left(x, \xi^{\prime}+\xi\right) h(y) b(y, \xi) d y d \xi^{\prime}
\end{aligned}
$$

We set

$$
r^{N}(x, \xi)=\sum_{|\alpha| \leq N} \frac{1}{\alpha !} a^{(\alpha)}(x, \xi) b_{(\alpha)}(x, \xi)
$$


We can see easily that there is a couple of constants $C_{0}$ and $C_{1}$ such that

$$
\sup _{x \in K}\left|D_{x}^{\beta} \partial_{\xi}^{\gamma} r^{N}(x, \xi)\right| \leq C_{0} C_{1}^{N+|\beta+\gamma|} N ! \gamma ! \beta !\left(1+|\xi|_{\varrho}\right)^{m-|\gamma|+\delta\left|\beta^{\prime}\right|}, \quad|\xi|_{\varrho} \geq B,
$$

where $m=m^{\prime}+m^{\prime \prime}$.

Theorem 3.3. Each $r^{N}(x, D)$ is an approximation of $r(x, D)$ in the following sense: We have

$$
r(x, D)-r^{N}(x, D)=F^{N}(x, D) \quad \text { in } \Omega^{\prime},
$$

where $F^{N}(x, D)$ can be written as a sum of two operators

$$
F^{N}(x, D)=F_{1}^{N}+F_{2}^{N}
$$

$F_{1}^{N}$ is an integral operator with the kernel $F_{1}^{N}(x, y) \in G^{\{\theta\}}\left(\Omega^{\prime} \times \Omega^{\prime}\right)$ and $F_{2}^{N}$ is a pseudodifferential operator with symbol $F_{2}^{N}(x, \xi)$ satisfying the condition

$$
\begin{aligned}
& \sup _{x \in \Omega^{\prime}}\left|D_{x}^{\beta} \partial_{\xi}^{\gamma} F_{2}^{N}(x, \xi)\right| \leq C_{0} C_{1}^{N+|\beta+\gamma|} N ! \gamma ! \beta !|\xi|_{\varrho}^{m_{+}+n^{\prime}-(1-\delta) N-|\gamma|} \\
& \cdot \sum_{\tau \leq \beta}\left(\begin{array}{c}
\beta \\
\tau
\end{array}\right)|\tau|^{\delta\left|\tau^{\prime}\right|}|\xi|_{\varrho}^{\delta\left|\beta^{\prime}-\tau^{\prime}\right|+\delta^{2}\left|\tau^{\prime}\right|} \\
&|\xi|_{\varrho} \geq B, m_{+}=\max (m, 0), \quad n^{\prime}=n^{\prime}(n) .
\end{aligned}
$$

Theorem 3.4. Let $a(x, \xi) \in S_{\varrho, \delta}^{m}\left(\Omega \times \mathbf{R}^{n}\right)$ and assume there are positive constants $c, B$ and $-\infty<m^{\prime}<\infty$ such that

$$
|a(x, \xi)| \geq c|\xi|_{\varrho}^{m^{\prime}}, \quad x \in \Omega, \quad|\xi|_{\varrho} \geq B .
$$

Assume also that for any compact set $K \subset \Omega$, there are positive constants $C_{0}$ and $C_{1}$ such that

$$
\begin{aligned}
\left|a_{(\beta)}^{(\alpha)}(x, \xi)\right| & \leq C_{0} C_{1}^{|\alpha+\beta|} \alpha ! \beta !|a(x, \xi)||\xi|_{\varrho}^{-|\alpha|+\delta\left|\beta^{\prime}\right|}, \\
x & \in K, \quad|\xi|_{\varrho} \geq B .
\end{aligned}
$$

Then the operator $a(x, D)$ is Gevrey hypoelliptic of order $\{\theta\}$ given in Theorem 3.2 .

Example 1. Take a differential operator considered in the paper [22]:

$$
P=x_{1}^{4}\left(\partial_{x_{2}}-\partial_{x_{1}}^{2}\right)+1, \quad\left(x_{1}, x_{2}\right) \in \mathbf{R}^{2} .
$$


Let $\Omega$ be small open neighborhood of $(0,0)$. We can see $n=2, p=1, \varrho_{1}=$ $1, \varrho_{2}=1 / 2, \delta=1 / 2$ and $\{\theta\}=\{2,2\}$ for the operator $P$ on $\Omega$. Optimality of this exponent is shown as follows. First take a function

$$
u_{0}\left(x_{1}, x_{2}\right)= \begin{cases}x_{1} e^{-\frac{1}{x_{1}}}, & x_{1}>0 \\ 0, & x_{1} \leq 0 .\end{cases}
$$

The function $u_{0}$ satisfies the equation $P u_{0}=0$ in $\mathbf{R}^{2}$ and it is well known the function $u_{0}$ is in $G^{\{2\}}(\Omega)$. Next take a solution $u_{1} \in G^{\{1,2\}}(\Omega)$ to the heat equation

$$
\left(\partial_{x_{2}}-\partial_{x_{1}}^{2}\right) u_{1}\left(x_{1}, x_{2}\right)=0 \text { in } \Omega .
$$

We seak a function $u$ such that

$$
P\left(u_{1}+u\right)=u_{1}+P u=0 \quad \text { in } \quad \Omega .
$$

We know adjoint operator ${ }^{t} P$ is also hypoelliptic so that such a function $u\left(x_{1}, x_{2}\right) \in G^{\{1,2\}}$ exists in $\Omega$ (shrinked if necessary) because of the solvability and Gevrey hypoellipticity of $P$.

\section{$\S 4$. Proof of Theorem 2.1: I, Gevrey Regularity in $x$}

For the proof of Gevrey hypoellipticity of the operator $P$ given in (2.4) with respect to the variable $\left(x_{1}, x_{2}, \ldots, x_{k}\right)$, we rely upon the method of pseudodifferential operators used in the paper [27] by making use of the preparation in Section 3. We introduce the notations

$$
\begin{aligned}
\langle\xi\rangle & =\left|\xi_{1}\right|^{\frac{1}{1+q_{1}}}+\left|\xi_{2}\right|^{\frac{1}{1+q_{2}}}+\cdots+\left|\xi_{k}\right|^{\frac{1}{1+q_{k}}} \\
|\xi|_{\varrho} & =\left|\xi_{1}\right|^{\frac{1+q_{k}}{1+q_{1}}}+\left|\xi_{2}\right|^{\frac{1+q_{k}}{1+q_{2}}}+\cdots+\left|\xi_{k}\right| \\
\left|x^{\prime}\right|_{\sigma} & =\left|x_{1}\right|^{\frac{1}{\sigma_{1}}}+\cdots+\left|x_{p}\right|^{\frac{1}{\sigma_{p}}}, \quad \varrho_{j}-\frac{1+q_{k}}{1+q_{j}}, j=1, \ldots, k .
\end{aligned}
$$

Under the conditions 1 through 3 on $P$, the following a priori estimate, called Grushin inequality, can be obtained:

Theorem 4.1 (cf. [12]). $\quad$ There exists a positive constant $C$ such that

$$
\begin{aligned}
& \sum_{|\beta| \leq m} \int\left|\left(\langle\xi\rangle+\left(\left|x^{\prime}\right|_{\sigma}+|y|\right)^{q_{1}}\left|\xi_{1}\right|+\cdots+\left(\left|x^{\prime}\right|_{\sigma}+|y|\right)^{q_{k}}\left|\xi_{k}\right|\right)^{m-|\beta|} D_{y}^{\beta} v(y)\right|^{2} d y \\
& \quad \leq C \int\left|P\left(x^{\prime}, y, \xi, D_{y}\right) v(y)\right|^{2} d y, \quad \xi \in \mathbf{R}^{k} \backslash\{0\}, \quad v \in C_{0}^{\infty}\left(\mathbf{R}_{y}^{n}\right) .
\end{aligned}
$$


We denote $P_{(\lambda)}^{(\mu)}\left(x^{\prime}, y, \xi, D_{y}\right)=\partial_{\xi}^{\mu} D_{x}^{\lambda} P\left(x^{\prime}, y, \xi, D_{y}\right), \mu, \lambda \in \mathbf{Z}_{+}^{k}$, as usual. Then we can derive the following estimates from (4.1).

Theorem 4.2 (cf. [12]). There are positive constants $C_{0}, C_{1}$ and $B$ such that

$$
\begin{aligned}
\left\|P_{(\lambda)}^{(\mu)}\left(x^{\prime}, y, \xi, D_{y}\right) v(y)\right\| & \leq C_{0} C_{1}^{|\mu+\lambda|} \mu ! \lambda !|\xi|_{\varrho}^{-|\mu|+\delta\left|\lambda^{\prime}\right|}\left\|P\left(x^{\prime}, y, \xi, D_{y}\right) v(y)\right\| \\
v & \in C_{0}^{\infty}\left(\mathbf{R}_{y}^{n}\right), \quad \mu, \lambda \in \mathbf{Z}_{+}^{k}, \quad|\xi|_{\varrho} \geq B
\end{aligned}
$$

Here $\|\cdot\|$ denotes the $L_{2}$-norm and $\delta=\sigma_{0} /\left(1+q_{k}\right)$ (see Section 2$)$.

Let $q$ be a positive rational number such that $q m$ is an integer. Then we denote by $H_{(m, q)}\left(\mathbf{R}_{y}^{n}\right)$ a weighted Sobolev space in $L_{2}\left(\mathbf{R}_{y}^{n}\right)$, equipped with the norm

$$
\|u\|_{H_{(m, q)}}=\left(\sum_{\substack{0 \leq|\gamma| \leq|m-| \beta \mid) \\ 0 \leq|\beta| \leq m}}\left\|y^{\gamma} D_{y}^{\beta} u\right\|_{L_{2}\left(\mathbf{R}^{n}\right)}^{2}\right)^{\frac{1}{2}} .
$$

We have the topological inclusion

$$
H_{(m, q)}\left(\mathbf{R}^{n}\right) \subset H_{m}\left(\mathbf{R}^{n}\right) \subset L_{2}\left(\mathbf{R}^{n}\right) .
$$

Theorem 4.3 (cf. [26], Theorems 3.3, 6.4, 7.4, [12] and [22]).

(i) Let $n \geq 2$ or $n=1$ and $\sigma \neq(0, \ldots, 0)$. Then there is the inverse $G\left(x^{\prime}, \xi\right) \in$ $\mathcal{L}\left(L_{2}\left(\mathbf{R}_{y}^{n}\right), H_{\left(m, q_{1}\right)}\left(\mathbf{R}_{y}^{n}\right)\right)$ of $P\left(x^{\prime}, y, \xi, D_{y}\right)$ such that

$$
\begin{aligned}
& G\left(x^{\prime}, \xi\right) P\left(x^{\prime}, y, \xi, D_{y}\right)=I \quad \text { in } \quad H_{\left(m, q_{1}\right)}\left(\mathbf{R}_{y}^{n}\right), \\
& P\left(x^{\prime}, y, \xi, D_{y}\right) G\left(x^{\prime}, \xi\right)=I \quad \text { in } \quad L_{2}\left(\mathbf{R}_{y}^{n}\right) .
\end{aligned}
$$

There are constants $C_{0}$ and $C_{1}$ such that

$$
\begin{aligned}
\left\|G_{(\lambda)}^{(\mu)}\right\|_{(m)} \leq & C_{0} C_{1}^{|\mu+\lambda|} \mu ! \lambda !|\xi|_{\varrho}^{-|\mu|+\delta\left|\lambda^{\prime}\right|}, \quad \mu, \lambda \in Z_{+}^{k}, \\
& \xi \in \mathbf{R}^{k} \backslash\{0\}, \quad|\xi|_{\varrho} \geq B .
\end{aligned}
$$

(ii) In case $n=1$ and $\sigma=(0, \ldots, 0)$, let $\Pi$ be the orthogonal projection on the null space of ${ }^{t} P\left(y, \xi, D_{y}\right)$. Then there is a pseudoinverse $G(\xi) \in \mathcal{L}\left(L_{2}\left(\mathbf{R}_{y}\right)\right.$, $\left.H_{\left(m, q_{1}\right)}\left(\mathbf{R}_{y}\right)\right)$ of $P\left(y, \xi, D_{y}\right)$ such that

$$
\begin{aligned}
& G(\xi) P\left(y, \xi, D_{y}\right)=I \quad \text { in } \quad H_{\left(m, q_{1}\right)}\left(\mathbf{R}_{y}\right), \\
& P\left(y, \xi, D_{y}\right) G(\xi)=I-\Pi(\xi) \quad \text { in } \quad L_{2}\left(\mathbf{R}_{y}\right) .
\end{aligned}
$$

There are constants $C_{0}$ and $C_{1}$ such that

$$
\left\|G^{(\mu)}(\xi)\right\|_{(m)} \leq C_{0} C_{1}^{|\mu|} \mu !|\xi|_{\varrho}^{-|\mu|}, \quad \mu \in Z_{+}^{k}, \quad|\xi|_{\varrho} \geq B .
$$


Now we can apply the results obtained in Section 3 and in the papers [12], [22] and [26]. Let $U$ be a small neighborhood of the origin of $\mathbf{R}_{x}^{k}$ and $B_{\mu}=\left\{y \in \mathbf{R}_{y}^{n} ;|y| \leq \mu\right\}$. Then starting with $G\left(x^{\prime}, D\right)$ we can construct an operator valued parametrix $\tilde{G}(x, D)$ of $Q(x, D) \equiv P\left(x^{\prime}, y, D_{x}, D_{y}\right)$ such that symbolically

$$
\tilde{G}(x, D) Q(x, D)=I+\mathcal{R} \quad \text { in } \quad C^{\infty}\left(U: L_{2}\left(B_{\mu}\right)\right)
$$

Here $\mathcal{R}$ is a regularizer in $\mathrm{x}$. In such a manner by using the method described in Section 3, (cf. [22]), we can show the Gevrey hypoellipticity in the $x$-direction.

Theorem 4.4. $\quad$ Let $P$ be the same operator as in Theorem 2.1 and consider the equation

$$
P\left(x^{\prime}, y, D_{x}, D_{y}\right) u(x, y)=f(x, y) \quad \text { in } \quad \Omega
$$

where $u(x, y) \in C^{\infty}(\Omega)$ and $f(x, y) \in G_{x, y}^{\{\theta, d\}}(\Omega)$, where $\theta=\left(\theta_{1}, \ldots, \theta_{k}\right)$ and $d=\left(\theta_{1}+k\right) /(1+k) \cdot I_{n}$ which are given in Section 2. Then we have $u \in G_{x}^{\{\theta\}}(\Omega)$.

\section{§5. Proof of Theorem 2.1: II, Gevrey Regularity in $y$; Bracket Calculus}

In this section, we shall prove the Gevrey hypoellipticity in the $y$-direction for the operators $P$ given in (2.4) in case $\sigma=0$, that is, for the operators of the third group by the classification in the paper [26]. In this case, the operator $P$ is written as follows:

$$
P\left(y, D_{y}, D_{x}\right)=\sum_{\substack{|\gamma|=\langle q, \alpha\rangle+|\alpha+\beta|-m \\|\alpha+\beta| \leq m}} a_{\alpha \beta \gamma} y^{\gamma} D_{x}^{\alpha} D_{y}^{\beta}
$$

Then we have $\delta=0$ and

$$
\begin{aligned}
\theta & =\left(\theta_{1}, \ldots, \theta_{j}, \ldots, \theta_{k}\right), \quad \theta_{j}=\frac{1+q_{j}}{1+q_{k}}, \quad j=1, \ldots, k, \\
\theta_{1} & \geq \theta_{2} \geq \cdots \geq \theta_{k}=1, \quad \theta_{1}>1, \\
d & =\frac{\theta_{1}+q_{1}}{1+q_{1}} \cdot I_{n}=\left(\frac{\theta_{1}+q_{1}}{1+q_{1}}, \ldots, \frac{\theta_{1}+q_{1}}{1+q_{1}}\right) .
\end{aligned}
$$

Let $\Omega$ be an open neighborhood of $(0,0) \in \mathbf{R}^{k+n}$ and consider the equation

$$
P\left(y, D_{x}, D_{y}\right) u(x, y)=f(x, y) \quad \text { in } \quad \Omega,
$$


where $u(x, y) \in C^{\infty}(\Omega) \cap G_{x}^{\{\theta\}}(\Omega)$ and $f \in G_{x, y}^{\{\theta, d\}}(\Omega)$. Then our purpose is to prove $u(x, y) \in G_{x, y}^{\{\theta, d\}}(\Omega)$.

In case $\sigma=0$, the estimate (4.1) yields the following one by Fourier transformation:

$$
\begin{aligned}
& \sum_{|\beta| \leq m} \int\left|\left(1+|y|^{q_{1}}\left|D_{x_{1}}\right|+\cdots+|y|^{q_{k}}\left|D_{x_{k}}\right|\right)^{m-|\beta|} D_{y}^{\beta} u(x, y)\right|^{2} d x d y \\
& \quad \leq C \int\left|P\left(y, D_{x}, D_{y}\right) u(x, y)\right|^{2} d x d y, \quad u \in C_{0}^{\infty}(\Omega)
\end{aligned}
$$

By an investigation of the quasi-homogeneity in $\xi$ and $y$, we have the following estimate with a positive constsnt $C=C(\Omega)$ :

$$
\left\|y^{\gamma} D_{x}^{\alpha-\tilde{\alpha}} D_{y}^{\beta+|\tilde{\alpha}|} u(x, y)\right\| \leq C\left\|P\left(y, D_{x}, D_{y}\right) u(x, y)\right\|, \quad u \in C_{0}^{\infty}(\Omega),
$$

where $|\gamma|=\langle q, \alpha\rangle+|\alpha|+|\beta|-m,|\alpha|+|\beta| \leq m$, and $0 \leq \tilde{\alpha} \leq \alpha$ and $D_{y}^{|\tilde{\alpha}|}$ denotes any derivative of the order $|\tilde{\alpha}|$ of $u$ with respect to $y$.

Furthermore, we can obtain the following estimate by applying the three line theorem of complex analysis, (cf. [20]).

Theorem 5.1. There exists a positive constant $C=C(\Omega)$ such that for any $\mu, 0<\mu<1$, we have

$$
\begin{aligned}
& \left\|\left(1+|y|^{q_{1}}\left|D_{x_{1}}\right|+\cdots+|y|^{q_{k}}\left|D_{x_{k}}\right|\right)^{m(1-\mu)}\left(1-\Delta_{y}\right)^{\frac{m}{2} \mu} u(x, y)\right\| \\
& \quad \leq C\left\|P\left(y, D_{x}, D_{y}\right) u(x, y)\right\|, \quad u \in C_{0}^{\infty}(\Omega) .
\end{aligned}
$$

Proof. Let us write $h=|y|^{q_{1}}\left|D_{x_{1}}\right|+\cdots+|y|^{q_{k}}\left|D_{x_{k}}\right|$. Then from the estimate (5.3) we have the following inequality:

$$
\begin{aligned}
\left\|(1+h)^{m} u(x, y)\right\|+\left\|\left(1-\Delta_{y}\right)^{\frac{m}{2}} u(x, y)\right\| & \leq C\left\|P\left(y, D_{x}, D_{y}\right) u(x, y)\right\|, \\
u(x, y) & \in C_{0}^{\infty}(\Omega) .
\end{aligned}
$$

For $v \in C_{0}^{\infty}\left(\mathbf{R}_{y}^{n}\right)$ consider an $L_{2}\left(\mathbf{R}_{y}^{n}\right)$-valued function

$$
f(z)=(1+h)^{m(1-z)}\left(1-\Delta_{y}\right)^{\frac{m}{2} z} v, \quad z \in \mathbf{C},
$$

Apparently $f(z)$ is holomorphic in $z \in \mathbf{C}$ and bounded in the strip $0 \leq$ $|\operatorname{Re}(z)| \leq 1$. By applying the three line theorem with any $\mu, 0<\mu<1$, we have

$$
\begin{aligned}
\|f(\mu)\| \leq & \sup _{\eta \in \mathbf{R}}\left\|(1+h)^{m(1-i \eta)}\left(1-\Delta_{y}\right)^{\frac{m}{2}(i \eta)} v\right\| \\
& +\sup _{\eta \in \mathbf{R}}\left\|(1+h)^{m(i \eta)}\left(1-\Delta_{y}\right)^{\frac{m}{2}(1+i \eta)} v\right\| \\
\leq & \left\|(1+h)^{m} v\right\|+\left\|\left(1-\Delta_{y}\right)^{\frac{m}{2}} v\right\| .
\end{aligned}
$$


We have the inequality

$$
\left\|(1+h)^{m(1-\mu)}\left(1-\Delta_{y}\right)^{\frac{m}{2} \mu} v\right\| \leq\left\|(1+h)^{m} v\right\|+\left\|\left(1-\Delta_{y}\right)^{\frac{m}{2}} v\right\| .
$$

Finally by applying Fourier transformation in $\xi$ and $x$, we get (5.5).

Now in Theorem 2.1, if $q_{1}=q_{2}=\cdots=q_{k}>0$ we have $\theta=(1,1, \ldots, 1)$ and $d=\left(1+q_{1}\right) /\left(1+q_{k}\right)=1$ and the operator $P$ is analytic hypoelliptic. In fact $P$ belongs to the first group by the classification in the paper [26] and there proved that it is analytic hypoelliptic in the space of hyperfunctions.

Therefore, we assume that $q_{1}>q_{k} \geq 0$ in the following. Then we have $1<d=\left(\theta_{1}+q_{1}\right) /\left(1+q_{k}\right)<\theta_{1}=\left(1+q_{1}\right) /\left(1+q_{k}\right)$. Thus, our purpose is to show Gevrey hypoellipticity of the operator $P$ with the exponent $d=\left(\theta_{1}+q_{1}\right) /\left(1+q_{k}\right)$ in $y=\left(y_{1}, y_{2}, \ldots, y_{n}\right)$.

Let us consider the equation

$$
P\left(y, D_{x}, D_{y}\right) u(x, y)=f(x, y) \quad \text { in } \quad \Omega,
$$

where $u(x, y) \in C^{\infty}(\Omega) \cap G_{x}^{\{\theta\}}(\Omega)$. Let $\omega$ be a small neighborhood of the origin such that $\bar{\omega} \subset \Omega$ and $\delta$ be a sufficiently small positive number. Then we can prepare a set of cut-off functions $\phi_{j}(x, y) \in C_{0}^{\infty}(\Omega)$ satisfying

$$
\begin{aligned}
\phi_{j} & \equiv 1 \quad \text { on } \quad \omega, \\
\nabla_{y} \phi_{j}(x, y) & \equiv 0, \quad|y| \leq \delta, \\
\left|D_{x, y}^{\alpha} \phi_{j}(x, y)\right| & \leq C_{0} C_{1}^{|\alpha|} j^{|\alpha|}, \quad|\alpha| \leq m j,
\end{aligned}
$$

where positive constants $C_{0}$ and $C_{1}$ are independent of $j=1,2, \ldots$, (cf. [15]).

We assume that the number $j$ is larger than $m$ and $m q_{1}$ and let $D_{y}^{j} u$ denote any derivative of the $j$-th order of $u$ in $y$. By the inequality (5.3) we have

$$
\left\|D_{y}^{m} \phi_{j} D_{y}^{j} u\right\| \leq C^{\prime}\left\|P \phi_{j} D_{y}^{j} u\right\| \leq C\left\{\left\|\phi_{j} D_{y}^{j} P u\right\|+\left\|\left[P, \phi_{j} D_{y}^{j}\right] u\right\|\right\} .
$$

By the assumption in Theorem 2.1 with respect to $f(x, y)$, the first term in the last side is estimated by the quantity of the form $C_{0} C_{1}^{j} j !^{d}$, where $d=$ $\left(\theta_{1}+q_{1}\right) /\left(1+q_{k}\right)$ and we have to investigate only the last term in the righthand side. For simplicity we denote by $\phi=\phi_{j}$ in the following. The last term in (5.6) consists of a linear sum of the terms

$$
\begin{aligned}
{\left[y^{\gamma} D_{x}^{\alpha} D_{y}^{\beta}, \phi D_{y}^{j}\right] u=} & \sum_{\substack{0 \leq \tilde{\alpha} \leq \alpha \\
0 \leq \tilde{\tilde{S}} \leq \beta \\
0<\tilde{\alpha}+\tilde{\beta}}} y^{\gamma}\left(\begin{array}{c}
\alpha \\
\tilde{\alpha}
\end{array}\right)\left(\begin{array}{c}
\beta \\
\tilde{\beta}
\end{array}\right)\left(D_{x}^{\tilde{\alpha}} D_{y}^{\tilde{\beta}} \phi\right) D_{x}^{\alpha-\tilde{\alpha}} D_{y}^{\beta-\tilde{\beta}} D_{y}^{j} u \\
& -\phi \sum_{0<\nu \leq \gamma}\left(\begin{array}{l}
j \\
\nu
\end{array}\right)\left(D_{y}^{\nu} y^{\gamma}\right) D_{x}^{\alpha} D_{y}^{\beta+j-\nu} u
\end{aligned}
$$


where $|\gamma|=\langle q, \alpha\rangle+|\alpha+\beta|-m$ and $|\alpha+\beta| \leq m$.

We can see that there is almost no problem with the first summation in the right-hand side. In fact, since the operator $P$ is uniformly elliptic for $|y| \geq \delta, L_{2}$-norm of the terms with $\tilde{\beta} \neq 0$ are estimated by the quantity of the kind $C_{0} C_{1}^{j} j !^{d}$, where the constants $C_{0}$ and $C_{1}$ are taken independent of $j$. We shall call such terms non-disturbing. Therefore, we need to investigate the $L^{2}$-norm of the terms with $\tilde{\beta}=0,0<\tilde{\alpha} \leq \alpha, 0 \leq|\beta|<m$ and $|\alpha+\beta| \leq m$ in the first summation:

$$
\left(\begin{array}{c}
\alpha \\
\tilde{\alpha}
\end{array}\right)\left\|y^{\gamma} D_{x}^{\tilde{\alpha}} \phi D_{x}^{\alpha-\tilde{\alpha}} D_{y}^{\beta} D_{y}^{j} u\right\| .
$$

First we shall consider the case where $\tilde{\alpha}=\alpha$. Denoting by $D_{y}^{|\alpha|}$ any derivative of the order $|\alpha|$ with respect to $y$ and so on, we see this is equal to

$$
\begin{aligned}
\left\|y^{\gamma} D_{x}^{\alpha} \phi \cdot D_{y}^{\beta} D_{y}^{j} u\right\| & =\left\|y^{\gamma} D_{x}^{\alpha} \phi \cdot D_{y}^{\beta+|\alpha|} D_{y}^{j-|\alpha|} u\right\| \\
& =\left\|y^{\gamma} D_{y}^{\beta+|\alpha|}\left(D_{x}^{\alpha} \phi \cdot D_{y}^{j-|\alpha|} u\right)\right\|+\text { non-disturbing terms. }
\end{aligned}
$$

By the estimates (5.4), we have

$$
\left\|y^{\gamma} D_{y}^{\beta+|\alpha|}\left(D_{x}^{\alpha} \phi \cdot D_{y}^{j-|\alpha|} u\right)\right\| \leq C\left\|P\left(D_{x}^{\alpha} \phi \cdot D_{y}^{j-|\alpha|} u\right)\right\|,
$$

where the constant $C$ can be taken independent of $j$ and $\alpha$. In the right-hand side of the above inequality, we see the order of the derivative in $y$ decreases: $D_{y}^{j} u \longrightarrow D_{y}^{j-|\alpha|} u$, and the same times of derivation of $\phi$ with respect to $x$ increases: $\phi \longrightarrow D_{x}^{\alpha} \phi$.

For each term with $0<\tilde{\alpha}<\alpha$, using a finite times (independent of $j$ ) of commutation, we see it is essentially (except non-disturbing terms) estimated by a constant times of

$$
\sum_{0<\tilde{\alpha}<\alpha}\left\|P\left(D_{x}^{\tilde{\alpha}} \phi \cdot D_{y}^{j-|\tilde{\alpha}|} u\right)\right\|
$$

by applying (5.3) and (5.4). By virtue of this procedure, we see $y$-derivation of $u$ decreases: $D_{y}^{j} u \longrightarrow D_{y}^{j-|\tilde{\alpha}|} u$. The same times of the derivation of $\phi$ with respect to $x$ increases: $\phi \longrightarrow D_{x}^{j-|\tilde{\alpha}|} \phi$. Continuing finite times (at most $j$ times) of these steps, we can see finally $L_{2}$-norm of the first summation in the right-hand side of (5.7) is estimated by the quantity of the kind $C_{0} C_{1}^{j} j !^{d}$, where $C_{0}$ and $C_{1}$ are independent of $j$.

It remains to treat the last summation in (5.7). We need to estimate the terms

$$
\begin{aligned}
& \left(\begin{array}{l}
j \\
\nu
\end{array}\right)\left\|\phi y^{\gamma-\nu} D_{x}^{\alpha} D_{y}^{\beta} D_{y}^{j-\nu} u\right\|, \quad 0<\nu \leq \gamma, \\
& |\gamma|=\langle q, \alpha\rangle+|\alpha+\beta|-m, \quad|\alpha+\beta| \leq m .
\end{aligned}
$$


First we consider the term with $\nu=\gamma$ :

$$
\begin{aligned}
\left(\begin{array}{c}
j \\
\gamma
\end{array}\right)\left\|\phi D_{x}^{\alpha} D_{y}^{\beta} D_{y}^{j-\gamma} u\right\| & =\left(\begin{array}{l}
j \\
\gamma
\end{array}\right)\left\|\phi D_{y}^{m} D_{y}^{\beta} D_{y}^{j-m-\gamma} D_{x}^{\alpha} u\right\| \\
& =\left(\begin{array}{l}
j \\
\gamma
\end{array}\right)\left\|D_{y}^{m}\left(\phi D_{y}^{j-m+\beta-\gamma} D_{x}^{\alpha} u\right)\right\|+\text { non-disturbing terms. }
\end{aligned}
$$

By the estimate (5.3), we have

$$
\left(\begin{array}{l}
j \\
\gamma
\end{array}\right)\left\|D_{y}^{m}\left(\phi D_{y}^{j-m+\beta-\gamma} D_{x}^{\alpha} u\right)\right\| \leq C\left(\begin{array}{l}
j \\
\gamma
\end{array}\right)\left\|P \phi D_{y}^{j-m+\beta-\gamma} D_{x}^{\alpha} u\right\| .
$$

We consider the procedure from (5.6) to (5.9) as a typical part of the first cycle of the total procedure (cf. [2]). That is, we started from (5.6) and we see that the order of derivative in $y$ of $u$ decreases with multiplication by $\left(\begin{array}{l}j \\ \gamma\end{array}\right)$ and the order of the derivative in $x$ of $u$ increases:

$$
D_{y}^{j} u \longrightarrow\left(\begin{array}{l}
j \\
\gamma
\end{array}\right) D_{y}^{j-(m-\beta+\gamma)} D_{x}^{\alpha} u \text {. }
$$

Since we have $\left(\begin{array}{l}j \\ \gamma\end{array}\right) \leq j^{|\gamma|}$, we may consider that, in such a typical cycle, for every loss of the power of $D_{y}$ there corresponds to the effect of multiplication of the kind

$$
j^{\frac{|\gamma|+\langle\theta, \alpha\rangle}{m-|\beta|+|\gamma|}} .
$$

By using the assumption $|\gamma|=\langle q, \alpha\rangle+|\alpha+\beta|-m \leq\langle q, \alpha\rangle$ and $\theta=(1+q) /(1+$ $\left.q_{k}\right)$, we can see such exponent of $j$ is always smaller than or equal to $d$ :

$$
\frac{|\gamma|+\langle\theta, \alpha\rangle}{m-|\beta|+|\gamma|} \leq \frac{\theta_{1}+q_{1}}{1+q_{1}}=d .
$$

It remains finally to investigate the terms with $0<\nu<\gamma$ in (5.8). By the cut-off function method, we may consider $u \in C_{0}^{\infty}(\Omega)$. We have

$$
\begin{aligned}
& j^{|\nu|}\left\|y^{\gamma-\nu} D_{x}^{\alpha} D_{y}^{\beta} D_{y}^{j-\nu} u(x, y)\right\| \\
& \leq j^{|\nu|}\left\|\left(|y|^{|\gamma|}\left|D_{x}^{\alpha}\right|\right)^{1-\frac{|\nu|}{|\gamma|}}\left|D_{x}^{\alpha}\right|^{\frac{|\nu|}{\gamma \mid}} D_{y}^{j+\beta-\nu} u(x, y)\right\| \\
& \leq j^{|\nu|}\left\|(1+h)^{(m-|\beta|)\left(1-\frac{|\nu|}{|\gamma|}\right)}\left|D_{x}^{\alpha}\right|^{\frac{|\nu|}{|\gamma|}} D_{y}^{j+\beta-\nu} u(x, y)\right\| \\
& =j^{|\nu|} \|(1+h)^{(m-|\beta|)\left(1-\frac{|\nu|}{|\gamma|}\right)}\left\langle D_{y}\right\rangle^{m \frac{|\nu|}{|\gamma|}+|\beta|\left(1-\frac{|\nu|}{|\gamma|}\right)} \\
& \text { - } D_{y}^{j+\beta-\nu}\left\langle D_{y}\right\rangle^{-m \frac{|\nu|}{|\gamma|}-|\beta|\left(1-\frac{|\nu|}{|\gamma|}\right)}\left|D_{x}^{\alpha}\right|^{\frac{|\nu|}{|\gamma|}} u(x, y) \| \\
& \leq C j^{|\nu|}\left\|P\left(y, D_{x}, D_{y}\right)\left\langle D_{y}\right\rangle^{j-\frac{|\nu|}{|\gamma|}(m-|\beta|+|\gamma|)}\left|D_{x}^{\alpha}\right|^{\frac{|\nu|}{\gamma \gamma}} u(x, y)\right\| .
\end{aligned}
$$

Here $\left\langle D_{y}\right\rangle=\left(1-\Delta_{y}\right)^{1 / 2}$ has only a symbolical meaning of the first-order derivation in $y$ and it may be justified and efficient at the end of the cycles. 
Thus, we may consider in this cycle for every loss of the power of $D_{y}$ there corresponds to the multiplication of the kind

$$
j^{\frac{|\nu|+\left\langle\theta, \alpha \frac{|\nu|}{\gamma \gamma}\right\rangle}{\mid \frac{\nu}{\gamma \mid}(m-|\beta|+|\gamma|)}} .
$$

Again we have the same inequality as before:

$$
\frac{|\nu|+\left\langle\theta, \alpha \frac{|\nu|}{|\gamma|}\right\rangle}{\frac{|\nu|}{|\gamma|}(m-|\beta|+|\gamma|)}=\frac{|\gamma|+\langle\theta, \alpha\rangle}{m-|\beta|+|\gamma|} \leq \frac{\theta_{1}+q_{1}}{1+q_{1}}=d .
$$

Thus, we can finally obtain the estimate of the form

$$
\left\|D_{y}^{m} \phi D_{y}^{j} u\right\|_{L_{2}(\omega)} \leq C_{0} C_{1}^{j} j^{j d}
$$

where $C_{0}$ and $C_{1}$ are independent of $j$.

\section{§6. Proof of Theorem 2.1: III, Gevrey Regularity in $y$; FBI-transformation}

It remains to determine the Gevrey exponent with respect to $y$-variables for the operators $P$ given in (2.4) in case $\sigma \neq 0$, that is, for the operators of the second group by the classification in the paper [26]. As was seen in Section 5 or in the paper [5], for those operators which hold the strong inequalities like (5.3) with $\sigma=0$ the method of bracket calculus is efficient, but it seems that it does not work well in case $\sigma \neq 0$. We shall apply the method of FBI-transformation used in the result of M. Christ [5], [6] etc. to overcome this difficulty.

At first we shall mention a non-isotropic version of the result by M. Christ, (cf. [5], Theorem 2.3). We refer to the paper [5] for the precise explanation of FBI-transformation.

We use the notation

$$
\langle\xi\rangle_{s}=\left(1+\xi_{1}^{2}\right)^{\frac{1}{2 s_{1}}}+\cdots+\left(1+\xi_{n}^{2}\right)^{\frac{1}{2 s_{n}}} .
$$

For $u(x) \in C_{0}^{\infty}\left(\mathbf{R}^{n}\right)$ and $(x, \xi) \in \mathbf{R}^{n} \times \mathbf{R}^{n}$, FBI-transformation of $u$ is defined by

$$
\mathcal{F}_{s} u(x, \xi)=\int u(y) e^{i\langle x-y, \xi\rangle-\langle\xi\rangle_{s}(x-y)^{2}} \alpha_{s}(x-y, \xi) d y
$$

where

$$
\alpha_{s}(x-y, \xi)=\prod_{j=1}^{n}\left(1+\frac{i}{s_{j}}\left(x_{j}-y_{j}\right) \xi_{j}\left(1+\xi_{j}^{2}\right)^{\frac{1}{2 s_{j}}-1}\right) .
$$


Then the following inversion formula holds:

$$
u(x)=(2 \pi)^{-n} \int \mathcal{F}_{s} u(x, \xi) d \xi, \quad u(x) \in C_{0}^{\infty}\left(\mathbf{R}^{n}\right) .
$$

Theorem 6.1 (cf. [5], Theorem 2.3). Let $s=\left(s_{1}, s_{2}, \ldots, s_{n}\right), s_{j} \geq 1$, $j=1,2, \ldots, n$, and $u(x) \in C_{0}^{\infty}\left(\mathbf{R}^{n}\right)$. Then the following four assertions are mutually equivalent:

(a) $u(x) \in G^{\{s\}}$ in a neighborhood of $x_{0} \in \mathbf{R}^{n}$.

(b) There exist $C, \delta \in \mathbf{R}_{+}$and a neighborhood $V$ of $x_{0}$ such that

$$
|\mathcal{F} u(x, \xi)| \leq C e^{-\delta \sum_{j=1}^{n}\left|\xi_{j}\right|^{\frac{1}{s_{j}}}} \cdot(x, \xi) \in V \times \mathbf{R}^{n},
$$

(c) There exist an open neighborhood $U=U\left(x_{0}\right) \subset \mathbf{C}^{n}$ of $x_{0}$ and $C, \delta \in \mathbf{R}_{+}$ such that, for each $\lambda \in \mathbf{R}_{+}^{n}, \sum \lambda_{j} \geq 1$, there exists a decomposition

$$
u=g_{\lambda}+h_{\lambda} \quad \text { in } \quad U \cap \mathbf{R}^{n}
$$

such that $g_{\lambda}$ is holomorphic in $U$,

$$
\left|g_{\lambda}(z)\right| \leq C e^{C|\operatorname{Im}\langle\lambda, z\rangle|}, \quad z \in U
$$

and

$$
\left|h_{\lambda}(x)\right| \leq C e^{-\delta \sum_{j=1}^{n} \lambda_{j}^{\frac{1}{s_{j}}}}, \quad x \in U \cap \mathbf{R}^{n} .
$$

(d) There exist an open neighborhood $U$ of $x_{0}$ and $C, \delta \in \mathbf{R}_{+}$such that for each $\lambda \in \mathbf{R}_{+}^{n}, \sum_{j=1}^{n} \lambda_{j} \geq 1$, there exists a decomposition

$$
u=g_{\lambda}+h_{\lambda} \quad \text { in } U \cap \mathbf{R}^{n}
$$

such that $g_{\lambda}$ is holomorphic in $\left\{z \in U:\left|\operatorname{Im}\left(z_{j}\right)\right| \leq \lambda_{j}^{\gamma_{j}-1}\right\}=U_{\lambda}$,

$$
\left|g_{\lambda}(z)\right| \leq C \quad z \in U_{\lambda},
$$

and

$$
\left|h_{\lambda}(x)\right| \leq C e^{-\delta \sum_{j=1}^{n} \lambda_{j}^{\frac{1}{s_{j}}}}, \quad x \in U \cap \mathbf{R}^{n} .
$$

Now we come back to consider the operator given in (2.4):

$$
P\left(x^{\prime}, y, D_{x}, D_{y}\right)=\sum_{\substack{\langle\sigma, \nu\rangle+|\gamma|=\langle q, \alpha\rangle+|\alpha+\beta|-m \\|\alpha+\beta| \leq m}} a_{\alpha \beta \nu \gamma} x^{\prime \nu} y^{\gamma} D_{x}^{\alpha} D_{y}^{\beta}, \quad a_{\alpha \beta \nu \gamma} \in \mathbf{C}
$$


We assume that $\sigma \neq 0$ under the same conditions given in Section 2. Let $\Omega$ be an open neighborhood of the origin $(0,0) \in \mathbf{R}_{x}^{k} \times \mathbf{R}_{y}^{n}$ and let $u, f \in C_{0}^{\infty}(\Omega)$ and we consider the equation

$$
P\left(x^{\prime}, y, D_{x}, D_{y}\right) u(x, y)=f(x, y) \quad \text { in } \quad \Omega .
$$

Our purpose is to prove $u$ is in $G_{x, y}^{\{\theta, d\}}$ in a neighborhood of $(0,0)$ if $f$ is so. By the result of Section 4, we may assume that $u(x, y)$ is already in $G_{x}^{\{\theta\}}$ in a neighborhood of $(0,0)$. Therefore, it is sufficient to prove that $u$ is in $G_{y}^{\{d\}}$ in a neighborhood of $(0,0)$, where $d=\left(\theta_{1}+q_{1}\right) /\left(1+q_{1}\right)$.

Let $(x, y),(\xi, \eta) \in \mathbf{R}^{k} \times \mathbf{R}^{n}$. We need to rewrite the definition of FBItransformation as follows:

$$
\begin{aligned}
\mathcal{F}_{\theta} u(x, y, \xi, \eta)= & \int u\left(x^{\prime}, y^{\prime}\right) e^{\left\{i\left(x-x^{\prime}\right) \xi+i\left(y-y^{\prime}\right) \eta-\langle\xi\rangle_{\theta}\left(x-x^{\prime}\right)^{2}-\langle\eta\rangle_{d}\left(y-y^{\prime}\right)^{2}\right\}} \\
& \cdot \alpha_{\theta}\left(x-x^{\prime}, y-y^{\prime}, \xi, \eta\right) d x^{\prime} d y^{\prime}
\end{aligned}
$$

where

$$
\begin{aligned}
\langle\xi\rangle_{\theta}= & \left(1+\xi_{1}^{2}\right)^{\frac{1}{2 \theta_{1}}}+\cdots+\left(1+\xi_{k}^{2}\right)^{\frac{1}{2 \theta_{k}}}, \\
\langle\eta\rangle_{d}= & \left(1+\eta_{1}^{2}+\cdots+\eta_{n}^{2}\right)^{\frac{1}{2 d}}, \quad d=\frac{\theta_{1}+q_{1}}{1+q_{1}}, \\
\alpha_{\theta}= & \prod_{j=1}^{k}\left(1+\frac{i}{\theta_{j}}\left(x_{j}-x_{j}^{\prime}\right) \xi_{j}\left(1+\xi_{j}^{2}\right)^{\frac{1}{2 \theta_{j}}-1}\right) \\
& \cdot \prod_{j=1}^{n}\left(1+\frac{i}{d}\left(y_{j}-y_{j}^{\prime}\right) \eta_{j}\left(1+\eta_{1}^{2}+\cdots+\eta_{n}^{2}\right)^{\frac{1}{2 d}-1}\right) .
\end{aligned}
$$

Then by applying Theorem 6.1 (b) for $\mathcal{F}_{\theta} u$, we can find an open neighborhood $V$ of $(0,0)$, and $C, \delta>0$ such that

$$
\begin{aligned}
\left|\mathcal{F}_{\theta} u(x, y, \xi, \eta)\right| & \leq C e^{-\delta \sum_{j=1}^{k}\left|\xi_{j}\right|^{\frac{1}{\theta_{j}}}} \leq C e^{-\delta|\xi|^{\frac{1}{\theta_{1}}}} \\
(x, y) & \in V, \quad(\xi, \eta) \in \mathbf{R}^{k} \times \mathbf{R}^{n} .
\end{aligned}
$$

Here we note that $\theta_{1} \geq \theta_{2} \geq \cdots \geq \theta_{k} \geq 0$ and $\theta_{1} \geq\left(1+q_{1}\right) /\left(1+q_{k}\right)>1$.

Let $c>0$ be a small constant determined later. Then from (6.4) we can find another couple of constants $C, \delta>0$ depending on $c$ such that

$$
\begin{gathered}
\left|\mathcal{F}_{\theta} u(x, y, \xi, \eta)\right| \leq C e^{-\delta\left(|\eta|^{\frac{1}{d}}+|\xi|^{\frac{1}{\theta_{1}}}\right)} \\
(x, y) \in V, \quad(\xi, \eta) \in \mathbf{R}^{k} \times \mathbf{R}^{n}, \quad c|\eta|^{\frac{1}{d}} \leq|\xi|^{\frac{1}{\theta_{1}}}
\end{gathered}
$$


Thus, the final problem left to prove is that we have the same type of the inequality as in (6.5) in the domain

$$
(x, y) \in V, \quad(\xi, \eta) \in \mathbf{R}^{k} \times \mathbf{R}^{n}, \quad c|\eta|^{\frac{1}{d}} \geq|\xi|^{\frac{1}{\theta_{1}}}
$$

for $V$ shrunk if necessary.

Now we use the notation like in [5]. We set

$$
E(x, y, \xi, \eta)=e^{i(\langle\tilde{x}-x, \xi\rangle+\langle\tilde{y}-y, \eta\rangle)-\langle\xi\rangle_{\theta}(\tilde{x}-x)^{2}-\langle\eta\rangle_{d}(\tilde{y}-y)^{2}}
$$

and

$$
\Psi(x, y, \xi, \eta)=\alpha_{\theta}(\tilde{x}-x, \tilde{y}-y, \xi, \eta) \cdot E(x, y, \xi, \eta),
$$

where $(\tilde{x}, \tilde{y}) \in V$ and $(\xi, \eta) \in \mathbf{R}^{k} \times \mathbf{R}^{n}$ are considered to be parameters.

Lemma 6.1. Let $P^{*}$ be the formal adjoint operator of $P$ :

$$
P^{*}=\sum_{\substack{\langle\sigma, \nu\rangle+|\gamma|=\{q, \alpha\rangle+|\alpha|+|\beta|-m \\|\alpha|+|\beta| \leq m}}(-1)^{|\alpha|+|\beta|} a_{\alpha \beta \nu \gamma} D_{x}^{\alpha} D_{y}^{\beta}\left(x^{\prime \nu} y^{\gamma}\right)
$$

Then there exist a small polydisk $D=\left\{y \in \mathbf{C}^{n} ;\left|y_{j}\right|<r\right\} \cup\left\{x \in \mathbf{C}^{k} ;\left|x_{j}\right|<r\right\}$ and $\delta, c>0$ such that for each $(\tilde{x}, \tilde{y}) \in D \cap \mathbf{R}^{k+n}$ and for each $(\xi, \eta) \in \mathbf{R}^{k} \times$ $\mathbf{R}^{n}, c|\eta|^{1 / d} \geq|\xi|^{1 / \theta_{1}}$, there exists $g \in C^{\infty}\left(D \cap \mathbf{R}^{k+n}\right)$ satisfying the following conditions:

$$
\begin{aligned}
P^{*}(E g) & =\Psi(x, y, \xi, \eta)+O\left(e^{-\delta\left(|\eta|^{\frac{1}{d}}+|\xi|^{\frac{1}{\theta_{1}}}\right.}\right), \\
(x, y) & \in D \cap \mathbf{R}^{k+n},
\end{aligned}
$$

where $g$ extends to a holomorphic function of $(x, y)$ and $g(x, y, \xi, \eta)=O(1)$ in

$$
U=D \cap\left\{|\operatorname{Im}(y)| \leq|\eta|^{\frac{1}{d}-1},\left|\operatorname{Im}\left(x_{j}\right)\right| \leq|\eta|^{\sigma_{j}\left(\frac{1}{d}-1\right)}\right\}, \quad c|\eta|^{\frac{1}{d}} \geq|\xi|^{\frac{1}{\theta_{1}}} .
$$

Before giving a proof of Lemma 6.1, we shall show how to use Lemma 6.1 to establish the inequality of the type (6.5) in the domain

$$
(x, y) \in V, \quad(\xi, \eta) \in \mathbf{R}^{k} \times \mathbf{R}^{n}, \quad c|\eta|^{\frac{1}{d}} \geq \sum_{j=1}^{k}\left|\xi_{j}\right|^{\frac{1}{\theta_{j}}},
$$

which completes the proof of Gevrey regularity of $u$ at $(0,0)$ by Theorem 6.1 and Theorem 4.4 .

We may suppose $u, f \in C_{0}^{\infty}(V)$ satisfying the equation (2.5) and $f \in G_{x, y}^{\{\theta, d\}}$ at $(0,0)$. Then we have

$$
\int_{V} P^{*} E g u(x, y) d x d y=\int_{V} E g P u d x d y=\int_{V} E g f(x, y) d x d y .
$$


On the other hand, by (6.7), this is equal to

$$
\begin{gathered}
\int \Psi(x, y) u(x, y) d x d y+O\left(e^{-\delta\left(|\eta|^{\frac{1}{d}}+|\xi|^{\frac{1}{\theta_{1}}}\right.}\right) \\
=\mathcal{F}_{\theta} u(\tilde{x}, \tilde{y}, \xi, \eta)+O\left(e^{-\delta\left(|\eta|^{\frac{1}{d}}+|\xi|^{\frac{1}{\theta_{1}}}\right.}\right) .
\end{gathered}
$$

By applying Theorem 6.1 (d) for $f(x, y)$, we can see that there exist a small complex neighborhood $U_{\xi, \eta}$ of $(0,0)$ and $\delta>0$ such that for each $(\xi, \eta) \in$ $\mathbf{R}^{k} \times \mathbf{R}^{n}$, there exists a decomposition

$$
f(x, y)=G(x, y, \xi, \eta)+O\left(e^{-\delta\left(|\eta|^{\frac{1}{d}}+|\xi|^{\frac{1}{\theta_{1}}}\right)}\right),
$$

where $G$ extends to a holomorphic function with respect to $y$ and $x$ and $O(1)$ in $U$. Of course we may assume that $U \cap \mathbf{R}^{k+n} \subset V$.

Now we have for all $(\tilde{x}, \tilde{y})$ in a compact subset of $V$

$$
\int_{V} E g f d x d y=\int_{V} E g G d x d y+O\left(e^{-\delta\left(|\eta|^{\frac{1}{d}}+|\xi|^{\frac{1}{\theta_{1}}}\right.}\right) .
$$

Let $r>0$ be a sufficiently small number and fix $\varphi \in C^{1}(\mathbf{R})$ such that $0 \leq \varphi \leq$ $1, \varphi=1$ for $|t| \leq r, \varphi=0$ for $|t| \geq 2 r$. Let $\varepsilon>0$ be small and shift the contour of integration

$$
y \longrightarrow \phi(y)=\left(y_{1}+i \varepsilon|\eta|^{\frac{1}{d}-1} \varphi\left(y_{1}\right), y_{2}, \ldots, y_{n}\right), \quad|\eta| \geq 1 .
$$

Then we obtain

$$
\begin{aligned}
\int_{V} E g G d x d y= & \int_{\mathbf{R}^{k+n}} e^{\left(i\langle\tilde{x}-x, \xi\rangle+i\langle\tilde{y}-\phi(y), \eta\rangle-\left(\langle\theta\rangle(\tilde{x}-x)^{2}+\langle\eta\rangle_{d}(\tilde{y}-\phi(y))^{2}\right)\right.} \\
& \cdot \alpha_{\theta}(\tilde{x}-x, \tilde{y}-\phi(y), \xi, \eta)\left(1+i \varepsilon|\eta|^{\frac{1}{d}-1} \varphi^{\prime}\left(y_{1}\right)\right) d x d y \\
= & O\left(e^{-\delta|\eta|^{\frac{1}{d}}}\right)=O\left(e^{-\delta^{\prime}\left(|\eta|^{\frac{1}{d}}+|\xi|^{\frac{1}{\theta_{1}}}\right.}\right)
\end{aligned}
$$

uniformly for

$$
(\xi, \eta) \in \mathbf{R}^{k+n}, \quad c|\eta|^{\frac{1}{d}} \geq|\xi|^{\frac{1}{\theta_{1}}}, \quad|\eta| \geq 1 .
$$

Proof of Lemma 6.1. Let $U \subset D \subset \mathbf{C}^{k+n}$ be the same type of the set given in Lemma 6.1 and define the space $H_{\infty}(U)$ of functions of $(x, y) \in U$ that are bounded and holomorphic with respect to $(x, y)$ in $D$. Here we consider $D$ a $2(k+n)$-dimensional measurable set.

Let

$$
E(x, y)=e^{i(\langle\tilde{x}-x, \xi\rangle+\langle\tilde{y}-y, \eta\rangle)-\langle\xi\rangle_{\theta}(\tilde{x}-x)^{2}-\langle\eta\rangle_{d}(\tilde{y}-y)^{2}}
$$


Then we write

$$
\begin{aligned}
E^{-1} P^{*} E= & \sum_{\substack{\langle\sigma, \nu\rangle+|\gamma|=\langle q, \alpha\rangle+|\alpha|+|\beta|-m \\
|\alpha|+|\beta| \leq m}}(-1)^{|\alpha|+|\beta|} \\
& \cdot\left\{a_{\alpha \beta \nu \gamma}\left(\xi+2\langle\xi\rangle_{\theta}(\tilde{x}-x)+D_{x}\right)^{\alpha}\left(\eta+2\langle\eta\rangle_{d}(\tilde{y}-y)+D_{y}\right)^{\beta}\right\}\left(x^{\prime} y^{\gamma}\right) \\
\equiv & \left(\sum_{|\beta|=m} a_{\beta}^{\prime} \eta^{\beta}+\sum_{\substack{\langle\sigma, \nu\rangle+|\gamma|=\langle q, \alpha\rangle \\
|\alpha|+|\beta|=m \\
|\beta|<m}} a_{\alpha \beta \nu \gamma} x^{\prime \nu} y^{\gamma} \xi^{\alpha} \eta^{\beta}\right)+\mathcal{R} \\
\equiv & \mathcal{A}+\mathcal{R},
\end{aligned}
$$

where $P^{*}$ is the operator given by $(6.6)$ which has the same properties as in $P$. We consider

$$
\mathcal{A}=\left(\sum_{|\beta|=m} a_{\beta}^{\prime} \eta^{\beta}+\sum_{\substack{\langle\sigma, \nu\rangle+|\gamma|=\langle q, \alpha\rangle \\|\alpha|+|\beta|=m \\|\beta|<m}} a_{\alpha \beta \nu \gamma} x^{\prime \nu} y^{\gamma} \xi^{\alpha} \eta^{\beta}\right)
$$

as a multiple operator from $H_{\infty}(U)$ to $H_{\infty}(U)$ and so on.

Lemma 6.2. Let $r=\operatorname{diamD}$ and $c>0$ be sufficiently small. Then $\mathcal{A}$ is considered to be an invertible operator from $H_{\infty}(U)$ to $H_{\infty}(U)$, where $U$ is given above.

Proof. By Conditions 1 and 2 given in Section 2, there is a positive constant $c_{0}$ such that

$$
\begin{gathered}
|\mathcal{A}| \geq c_{0}\left(|\eta|+\left(\left|x^{\prime}\right|_{\sigma}+|y|\right)^{q_{1}}\left|\xi_{1}\right|+\cdots+\left(\left|x^{\prime}\right|_{\sigma}+|y|\right)^{q_{k}}\left|\xi_{k}\right|\right)^{m} \geq c_{0}|\eta|^{m} \\
(x, y) \in \mathbf{R}^{k+n}, \quad(\xi, \eta) \in \mathbf{R}^{k+n}, \quad|\eta| \geq 1
\end{gathered}
$$

where

$$
\left|x^{\prime}\right|_{\sigma}=\left|x_{1}\right|^{\frac{1}{\sigma_{1}}}+\cdots+\left|x_{p}\right|^{\frac{1}{\sigma_{p}}}
$$

as was given in Section 4 .

Next, for $(x, y) \in \mathbf{C}^{k+n}$, considering the method of quasi-homogeneity, we have

$$
\begin{gathered}
|\mathcal{A}| \geq c_{0}{ }^{\prime}\left(|\eta|+\left(\left|x^{\prime}\right|_{\sigma}+|\operatorname{Re}(y)|\right)^{q_{1}}\left|\xi_{1}\right|+\cdots+\left(\left|x^{\prime}\right|_{\sigma}+|\operatorname{Re}(y)|\right)^{q_{k}}\left|\xi_{k}\right|\right)^{m} \\
-c_{0}{ }^{\prime \prime}\left(|\operatorname{Im}(y)|^{q_{1}}\left|\xi_{1}\right|+\cdots+|\operatorname{Im}(y)|^{q_{k}}\left|\xi_{k}\right|\right)^{m}
\end{gathered}
$$


for some constants $c_{0}{ }^{\prime}, c_{0}{ }^{\prime \prime}>0$.

Now for any $\varepsilon>0$, we use the assumption $\left|\operatorname{Im}\left(x_{j}\right)\right| \leq|\eta|^{\sigma_{j}((1 / d)-1)},|\operatorname{Im}(y)|$ $\leq|\eta|^{(1 / d)-1}$ and $c|\eta|^{1 / d} \geq \sum_{j=1}^{k}\left|\xi_{j}\right|^{1 / \theta_{j}},|\eta| \geq 1$, to obtain the estimate of the form

$$
\left(\left|\operatorname{Im}\left(x^{\prime}\right)\right|_{\sigma}+|\operatorname{Im}(y)|\right)^{q_{j}}\left|\xi_{j}\right| \leq \varepsilon|\eta|, \quad j=1, \ldots, k,
$$

if the constant $c$ is taken sufficiently small. Thus we have with some $c_{0}>0$ the inequality

$$
\begin{gathered}
|\mathcal{A}| \geq c_{0}\left(|\eta|+\left(\left|\operatorname{Re}\left(x^{\prime}\right)\right|_{\sigma}+|\operatorname{Re}(y)|\right)^{q_{1}}\left|\xi_{1}\right|+\cdots+\left(\left|\operatorname{Re}\left(x^{\prime}\right)\right|_{\sigma}+|\operatorname{Re}(y)|\right)^{q_{k}}\left|\xi_{k}\right|\right)^{m} \\
(x, y) \in U, \quad c|\eta|^{\frac{1}{d}} \geq \sum_{j=1}^{k}\left|\xi_{j}\right|^{\frac{1}{\theta_{j}}}, \quad|\eta| \geq 1 .
\end{gathered}
$$

Lemma 6.3 (cf. [5]). Let $D^{\prime} \subset D$ be bounded open domains in $\mathbf{C}_{z}$ with distance $\left(D^{\prime}, \partial D\right) \geq \varepsilon>0$. Then the norm of the operator

$$
D_{z}: H_{\infty}(D) \longrightarrow H_{\infty}\left(D^{\prime}\right)
$$

is $O\left(\varepsilon^{-1}\right)$.

We omit the proof which is obtained by using Cauchy's integral formula.

Let $D_{1}$ and $D_{\infty}$ be the open polydisks with center at $(0,0)$ and diam $D_{1}=r$ and $\operatorname{diam} D_{\infty}=(1 / 2) r$. We write

$$
\begin{gathered}
U_{1}=D_{1} \cap\left\{|\operatorname{Im}(y)| \leq|\eta|^{\frac{1}{d}-1},\left|\operatorname{Im}\left(x_{j}\right)\right| \leq|\eta|^{\sigma_{j}\left(\frac{1}{d}-1\right)} \text { if } \quad \sigma_{j} \geq 1 \quad\right. \text { or } \\
\left.\quad\left|\operatorname{Im}\left(x_{j}\right)\right| \leq|\eta|^{\frac{1}{d}-1} \quad \text { if } \quad \sigma_{j}<1, c|\eta|^{\frac{1}{d}} \geq \sum_{j=1}^{k}\left|\xi_{j}\right|^{\frac{1}{\theta_{j}}}\right\}, \\
U_{\infty}=D_{\infty} \cap\left\{|\operatorname{Im}(y)| \leq \frac{1}{2}|\eta|^{\frac{1}{d}-1},\left|\operatorname{Im}\left(x_{j}\right)\right| \leq \frac{1}{2}|\eta|^{\sigma_{j}\left(\frac{1}{d}-1\right)} \quad \text { if } \quad \sigma_{j} \geq 1 \quad\right. \text { or } \\
\left.\left|\operatorname{Im}\left(x_{j}\right)\right| \leq \frac{1}{2}|\eta|^{\frac{1}{d}-1} \quad \text { if } \quad \sigma_{j} \leq 1, c|\eta|^{\frac{1}{d}} \geq \sum_{j=1}^{k}\left|\xi_{j}\right|^{\frac{1}{\theta_{j}}}\right\} .
\end{gathered}
$$

Let $\Lambda>0$ be a large constant to be chosen later. Given a large $\eta$, choose an integer $N=\left[\Lambda^{-1}|\eta|^{1 / d}\right]$, where $[a]$ denotes the largest integer less than or equal to $a$. For $2 \leq j \leq m N$ construct open sets $U_{\infty}=U_{m N} \subset \subset U_{m N-1} \subset \subset$ $\cdots \subset \subset U_{1}$ satisfying

$$
\operatorname{distance}\left(\left.U_{j+1}\right|_{\mathbf{C}_{y}^{k}},\left.\partial U_{j}\right|_{\mathbf{C}_{y}^{k}}\right) \geq \varepsilon \Lambda|\eta|^{-1},
$$




$$
\operatorname{distance}\left(\left.U_{j+1}\right|_{x_{l}},\left.\partial U_{j}\right|_{x_{l}}\right) \geq \varepsilon \Lambda|\eta|^{-\sigma_{l}} \quad \text { if } \quad \sigma_{l} \geq 1, \varepsilon \Lambda|\eta|^{-1} \quad \text { if } \quad \sigma_{l} \leq 1 .
$$

Here $\left.U_{j}\right|_{\mathbf{C}_{y}^{k}}=U_{j} \cap \mathbf{C}_{y}^{k}$, etc. and $\varepsilon$ is a small constant, independent of $\eta, \Lambda, j$. This is possible because for large $\eta$ we have

$$
1 \leq \sigma_{j} \Longrightarrow|\eta|^{\frac{1}{d}-\sigma_{j}} \leq\left.\eta\right|^{\sigma_{j}\left(\frac{1}{d}-1\right)} .
$$

Now we can complete the proof of Lemma 6.1. Considering $\mathcal{R}$ an operator from $H_{\infty}\left(U_{j}\right)$ to $H_{\infty}\left(U_{j+m}\right)$, we assume that $\mathcal{A}^{-1} \mathcal{R}$ is sufficiently small for the moment. In order to solve the equation $(\mathcal{A}+\mathcal{R}) g=\alpha$, we define

$$
g=\sum_{j=0}^{N}(-1)^{j}\left(\mathcal{A}^{-1} \mathcal{R}\right)^{j} \mathcal{A}^{-1} \alpha
$$

Thus we have

$$
\begin{aligned}
(\mathcal{A}+\mathcal{R}) g & =\alpha+(-1)^{N+1}\left(\mathcal{R} \mathcal{A}^{-1}\right)^{N+1} \alpha \\
\left(\mathcal{R} \mathcal{A}^{-1}\right)^{N+1} & =O(\exp (-\varepsilon N))=O\left(\exp \left(-\varepsilon^{\prime}|\eta|^{\frac{1}{d}}\right)\right) .
\end{aligned}
$$

From where we have the estimation of the form (6.7) in the domain $U_{\infty}$.

It remains to estimate $\mathcal{A}^{-1} \mathcal{R}$ in the sense of a linear operator from $H_{\infty}\left(U_{j}\right)$ to $H_{\infty}\left(U_{j+m}\right)$. We can see the inequality (6.10) holds for $(x, y) \in U_{1}$. Therefore with some positive constant $C$, we have the following estimation in $U_{1}$ :

$$
\left|\mathcal{A}^{-1} \mathcal{R}\right| \leq \frac{C|\mathcal{R}|}{\left(|\eta|+\left(\left|x^{\prime}\right|_{\sigma}+|y|\right)^{q_{1}}\left|\xi_{1}\right|+\cdots+\left(\left|x^{\prime}\right|_{\sigma}+|y|\right)^{q_{k}}\left|\xi_{k}\right|\right)^{m}} .
$$

The right-hand side is composed of the terms of the form

$$
\begin{gathered}
\frac{\xi^{\alpha_{1}}\left(\langle\xi\rangle_{\theta}(\tilde{x}-x)\right)^{\alpha_{2}} x^{\prime \nu-\alpha_{3}} D_{x}^{\alpha_{4}} \cdot \eta^{\beta_{1}}\left(\langle\eta\rangle_{d}(\tilde{y}-y)\right)^{\beta_{2}} y^{\gamma-\beta_{3}} D_{y}^{\beta_{4}}}{\left(|\eta|+\left(\left|x^{\prime}\right|_{\sigma}+|y|\right)^{q_{1}}\left|\xi_{1}\right|+\cdots+\left(\left|x^{\prime}\right|_{\sigma}+|y|\right)^{q_{k}}\left|\xi_{k}\right|\right)^{m}}, \\
\alpha=\alpha_{1}+\alpha_{2}+\alpha_{3}+\alpha_{4}, \quad\left|\alpha_{2}+\alpha_{3}+\alpha_{4}\right|>0 \quad \text { if } \quad \alpha>0, \\
\beta=\beta_{1}+\beta_{2}+\beta_{3}+\beta_{4}, \quad\left|\beta_{2}+\beta_{3}+\beta_{4}\right|>0 \quad \text { if } \quad \beta>0, \quad|\alpha+\beta| \leq m, \\
\langle\sigma, \nu\rangle+|\gamma|=\langle q, \alpha\rangle+|\alpha+\beta|-m .
\end{gathered}
$$

The quasi-homogeneous order (cf. the condition 1 given in Section 2) of the denominator is $m$. In the numerator, $D_{x}$ and $D_{y}$ are operators with norms $O\left(\Lambda^{-1}|\eta|^{\sigma_{j}}\right)$ and $O\left(\Lambda^{-1}|\eta|\right)$. Hence we may consider the quasi-homogeneous orders of them are $\sigma_{j}$ or 1 . The terms $\langle\xi\rangle_{\theta}(\tilde{x}-x)$ and $\langle\eta\rangle_{d}(\tilde{y}-y)$ are estimated by $r|\eta|$, hence we may also consider the quasi-homogeneous orders of them are 
1. Thus with parameter $\lambda>0$, we may consider that the quasi-homogeneous order of (6.12) is estimated by

$$
O\left(\lambda^{-\left\langle q, \alpha_{2}\right\rangle-\left\langle 1+q-\sigma, \alpha_{3}\right\rangle-\left\langle 1+q-\tilde{\sigma}, \alpha_{4}\right\rangle}\right) \cdot O\left(\Lambda^{-\left|\alpha_{4}\right|} \cdot r^{\left|\alpha_{2}\right|}\right)
$$

where

$$
\tilde{\sigma}=\left(\tilde{\sigma_{1}}, \ldots, \tilde{\sigma_{p}}\right), \tilde{\sigma_{j}}=\sigma_{j} \quad \text { if } \quad \sigma_{j} \geq 1 \quad \text { and } \quad \tilde{\sigma_{j}}=1 \quad \text { if } \quad \sigma_{j}<1 .
$$

The hypothesis $1+q_{k}>\max \left(\sigma_{1}, \ldots, \sigma_{p}\right)$ given in Section 2 with the condition $\left|\alpha_{2}+\alpha_{3}+\alpha_{4}\right|>0$ (if $\alpha>0$ ) assures the negative order of the above quantity, and finally we have

$$
\left|\mathcal{A}^{-1} \mathcal{R}\right|<1
$$

in the sense of a linear operator from $H_{\infty}\left(U_{j}\right)$ to $H_{\infty}\left(U_{j+m}\right)$ if $c$ and $r$ are taken sufficiently small and $\Lambda$ and $|\eta|$ sufficiently large.

\section{References}

[1] Baouendi, M. S. and Goulaouic, C., Nonanalytic-hypoellipticity for some degenerate elliptic operators, Bull. Amer. Math. Soc., 78 (1972), 483-486.

[2] Bove, A. and Tartakoff, D., Optimal non-isotropic Gevrey exponents for sums of squares of vector fields, Comm. Partial Differential Equations, 22 (1997), 1263-1282.

[3] Bros, J. and Iagolnizter, D., Support essentiel et structure analytique, Séminaire Goulaouic-Lions-Schwartz 1975-1976, Éxposé No.18.

[4] Chen, Dong and Matsuzawa, T., $S$-spaces of Gel'fand-Shilov and differential equations, Japan. J. Math., 92 (1994), 227-239.

[5] Christ, M., Intermediate optimal Gevrey exponents occur, Comm. Partial Differential Equations, 22 (1997), 359-379.

[6] - Examples pertaining to Gevrey hypoellipticity, Math. Res. Lett., 4 (1997), 725733.

[7] Derridi, M. and Zuily, C., Régularité analytique et Gevrey pour des classes d'opérateurs élliptiques paraboliques dégénérés du second ordre, Astérisque, 2, 3 (1973), 371-381.

[8] Gel'fand, I. M. and Shilov, G. E., Generalized Functions, Vol.2 and Vol.3, Academic Press, New York-London, 1968.

[9] Grushin, V. V., Pseudodifferential operators on $R^{n}$ with bounded symmbols, Funct. Anal. Appl., 4 (1970), 202-212.

[10] - On a class of hypoelliptic operators, Math. USSR Sbornik, 12 (1970), 458-476.

[11] - On a class of elliptic pseudodifferential operators degenerate on a submanifold, Math. USSR Sbornik, 13 (1971), 155-185.

[12] - Hypoelliptic differential equations and pseudodifferential operators with operator-valued symmbols, Math. USSR Sbornik, 17 (1972), 497-514.

[13] Hanges, N. and Himonas, A. A., Singular solutions for sums of squares of vector fields, Comm. Partial Differential Equations, 16 (1991), 1503-1511.

[14] Hashimoto, S., Matsuzawa, T. and Morimoto, Y., Opérateurs pseudo-differentiels et classes de Gevrey, Comm. Partial Differential Equations, 8 (12) (1983), 1277-1289.

[15] Hörmander, L., Linear Partial Differential Operators, Springer Verlag, BerlinGöttingen-Heidelberg, 1963. 
[16] Hörmander, L., Pseudodifferential operators and hypoelliptic equations, Amer. Math. Soc. Symp. on Singular Integrals, (1966), 138-183.

[17] - Hypoellipic second order differential equations, Acta Math., 119 (1967), 147171.

[18] - The Analysis of Linear Partial Differential Operators 1, Springer Verlag, BerlinHeidelberg-New York-Tokyo, 1983.

[19] Krantz, S. G. and Parks, H. R., A Primer of Real Analytic Functions, Birkhäuser Verlag, Basel-Boston-Berlin, 1992.

[20] Lions, J. L. and Magenes, E., Problèmes aux Limites non homogènes et Applications, Vol.1, Dunod, 1968.

[21] Matsuzawa, T., Sur les équations $u_{t t}+t^{\alpha} u_{x x}=f(\alpha \geq 0)$, Nagoya Math. J., 42 (1971), 43-55.

[22] - Gevrey hypoellipticity of pseudodifferential operators, Tôhoku Math. J., 39 (1987), 447-464.

[23] - A calculus approach to hyperfunctions I, Nagoya Math. J., 108 (1987), 53-66.

[24] A calculus approach to hyperfunctions II, Trans. Amer. Math. Soc., 313 (1989), 619-654.

[25] - Foundation of a calculus approach to hyperfunctions and applications, Monograph, 1995.

[26] - Gevrey hypoellipticity for Grushin operators, Publ. RIMS, Kyoto Univ., 33 (1997), 775-799.

[27] $\_$An Introduction to the Theory of Partial Differential Equations, JSPS-DOST Lecture Notes in Math., 4, Sophia University, 1997.

[28] — Optimal Gevrey exponents for some degenerate elliptic operators, J. Korean Math. Soc., 35 (1998), 981-997.

[29] Métivier, G., Non-hypoellipticité analytique pour $D_{x}^{2}+\left(x^{2}+y^{2}\right) D_{y}^{2}, C$. R. Acad. Sci. Paris, Sér. 1, 292 (1981), 401-404.

[30] - Analytic hypoellipticity for operators with multiple characteristics, Comm. Partial Differential Equations, 6 (1981), 1-90.

[31] Okaji, T., Analytic hypoellipticity for operators with symplectic characteristics, J. Math. Kyoto Univ., 25 (1985), 489-514.

[32] Oleinik, O. and Radkevich, R., On the analyticity of solutions to partial differential equations and systems, Astérisque, 2, 3 (1973), 272-285.

[33] Rodino, L., Linear Partial Differential Operators in Gevrey Spaces, World Scientific, Singapore-New Jersey-London-Hong Kong, 1993.

[34] Sjöstrand, J., Propagation of analytic singularities for second order Dirichlet problems, Comm. Partial Differential Equations, 5 (1) (1980), 41-94.

[35] Treves, F., Introduction to Pseudodifferential and Fourier Integral Opetators, 1 and 2, Plenum Press, New York-London, 1980.

[36] - Symplectic geometry and analytic hypoellipticity, Differential equations; La Pietra 1996 (Florence), 201-219, Proc. Sympos. Pure Math., 65, Amer. Math. Soc., Providence, RI, 1999 